\title{
1 In vivo identification and validation of novel potential predictors for human
}

\section{2 cardiovascular diseases}

3

4 Omar T. Hammouda ${ }^{1,2}$, Meng Yue $\mathrm{Wu}^{1}$, Verena Kaul ${ }^{1}$, Jakob Gierten ${ }^{1,2,3}$, Thomas

5 Thumberger ${ }^{1}$ and Joachim Wittbrodt ${ }^{1, *}$

6

$7 \quad{ }^{1}$ Centre for Organismal Studies Heidelberg, Heidelberg University, Im Neuenheimer Feld 230,

869120 Heidelberg, Germany

9 2 Heidelberg Biosciences International Graduate School, Heidelberg University, Im

10 Neuenheimer Feld 501, 69120 Heidelberg, Germany

$11{ }^{3}$ Department of Pediatric Cardiology and Congenital Heart Diseases, University Hospital

12 Heidelberg, Im Neuenheimer Feld 430, 69120 Heidelberg, Germany

$13{ }^{*}$ Corresponding Author

14

15 Running title (40 char): Validating understudied GWAS heart genes

16

17

18

19 Final character count (with spaces): 21,783 


\section{Abstract}

Genetics crucially contributes to cardiovascular diseases (CVDs), the global leading cause of death. Since the majority of CVDs can be prevented by early intervention there is a high demand

24 for predictive markers. While genome wide association studies (GWAS) correlate genes and CVDs after diagnosis and provide a valuable resource for such markers, preferentially those with previously known or suspected function are addressed further. To tackle the unaddressed blind spot of understudied genes, we particularly focused on the validation of heart GWAS candidates with little or no apparent connection to cardiac function. Building on the high conservation of basic heart function and underlying genetics from fish to human we combined CRISPR/Cas9 genome editing of the orthologs of human GWAS candidates in isogenic medaka with automated high-throughput heart rate analysis. Our functional analyses of understudied human candidates uncovered a prominent fraction of heart rate associated genes from adult human patients displaying a heart rate effect in embryonic medaka already in the injected generation. Following this pipeline, we identified 16 GWAS candidates with potential diagnostic and predictive power for human CVDs.

Keywords: CRISPR / gene validation / GWAS / heart rate / high throughput analysis 


\section{Introduction}

41 Genetics crucially contributes to the development and progression of cardiovascular diseases (CVDs), the global leading cause of death (Kathiresan \& Srivastava 2012; Cambien \& Tiret 2007). Elevated resting heart rate in humans has been widely considered as a potential, modifiable risk factor of cardiovascular and all-cause mortality (Beere et al. 1984; DYER et al. 1980; MD et al. 2010; Gillum et al. 1991). Since the majority of CVDs can be prevented by early intervention (McGill et al. 2008) there is a high demand for diagnostic and predictive CVD markers. Genome wide association studies (GWAS) on human patients correlate genes and CVDs after diagnosis and provide a valuable resource for those putative markers (Eicher et al. 2015). However, genes with pre-assigned cardiac functions are usually more likely to be addressed further, while uncharacterized genes or those with no pre-existing evidence to the heart are often neglected. This is likely due to the lack of experimental pipelines for the rapid and robust validation of such markers with implications for heart function. Recently, we demonstrated the power of targeted genome editing in the small animal model system medaka (Oryzias latipes) to validate trabeculation-associated genes (Meyer et al. 2020). The ease of manipulation combined with robust acquisition and analysis pipelines highlight the power of using fish embryos in high-throughput applications (Gierten et al. 2020; Shankaran et al. 2018; Lessman 2011; Oxendine et al. 2006). Embryos of fish model systems undergo extrauterine development in a transparent egg. This allows to monitor heart development and heart rate noninvasively in live undisturbed embryos for an extended period of time. Heart development, function and physiology in fish, though simpler, is comparable to mammals (Nemtsas et al. 2010; Yonekura et al. 2018; Gut et al. 2017). Here we combined targeted genome editing via CRISPR/Cas9 (Stemmer et al. 2015; Thumberger et al. 2021) with automated high-throughput imaging and heart rate analysis in isogenic medaka embryos (Gierten et al. 2020) to enable functional analyses directly in the injected generation (F0). We tested the performance our assay with a positive control ( $n k x 2-5)$, evaluated the random discovery rate and analyzed 40 heart associated genes identified from human GWAS. Our assay uncovered that $57 \%$ of candidates assigned to human heart rate in GWAS also affected heart rate in fish embryos. We 
have thus experimentally validated understudied human GWAS candidates, identifying 16 genes with potential diagnostic and predictive power for human CVDs.

\section{Results}

For the straight forward functional validation of GWAS candidates we aimed at combining CRISPR/Cas9-mediated targeted gene inactivation in the injected generation of medaka embryos with high content screening approaches to validate the impact of the loss-of-function on the heart rate (Fig 1A). We first assessed the efficiency of the CRISPR/Cas9 system by targeting the green fluorescent protein $(g f p)$ gene in a transgenic medaka reporter line expressing GFP and mCherry fluorescent proteins exclusively in the heart via the cardiac myosin light chain 2 ( $\mathrm{cmlc} 2$ ) promoter. Injection of the heiCas 9 mRNA, i.e. a Cas 9 equipped with an early active nuclear localization signal (Thumberger et al. 2021), together with a guide RNA targeting $g f p$ into medaka embryos at the 1-cell stage resulted in a complete loss of GFP expression in the heart ( $\mathrm{n}=8 / 8$ ) (Fig 1B and Fig S1). Only when injected into a single blastomere of the four-cell stage or later, a mosaic pattern was observable. This demonstrates the high efficiency of our approach already in the injected generation.

For our functional validation assay, we used the cardiac-specific homeobox-containing transcription factor NKX2-5 as a positive control. In human patients, a single amino acid mutation in the homeodomain (R141C) was previously associated with atrial septal defect (ASD) and shown to cause delayed heart morphogenesis in adult mice (Zakariyah et al. 2017). To test our high-throughput imaging and heart rate analysis pipeline for functional in vivo gene validation in the injected generation, we targeted the region orthologous to R141C in medaka embryos using the CRISPR/Cas9 system (Stemmer et al. 2015, Thumberger et al. 2021). As a negative control, we targeted the oculocutaneous albinism 2 locus (oca2) (Fukamachi et al. 2004) as a heart-unrelated pigmentation gene. The apparent loss of the eye pigmentation phenotype (and low degree of mosaicism) (Lischik et al. 2019; Hammouda et al. 2019) underscores the bi-allelic knock-out efficacy of our system. Injections into wild-type medaka embryos were performed at the 1-cell stage, and hereafter resulting embryos are referred to as crispants. To address the impact on the heart rate we raised the medaka crispants until cardiac 
bioRxiv preprint doi: https://doi.org/10.1101/2021.02.03.429563; this version posted June 21, 2021. The copyright holder for this preprint (which was not certified by peer review) is the author/funder, who has granted bioRxiv a license to display the preprint in perpetuity. It is made available under aCC-BY-NC-ND 4.0 International license.

A Gene targeting

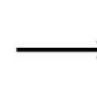

High-throughput crispant embryo imaging

Crispant embryo heart rate analysis
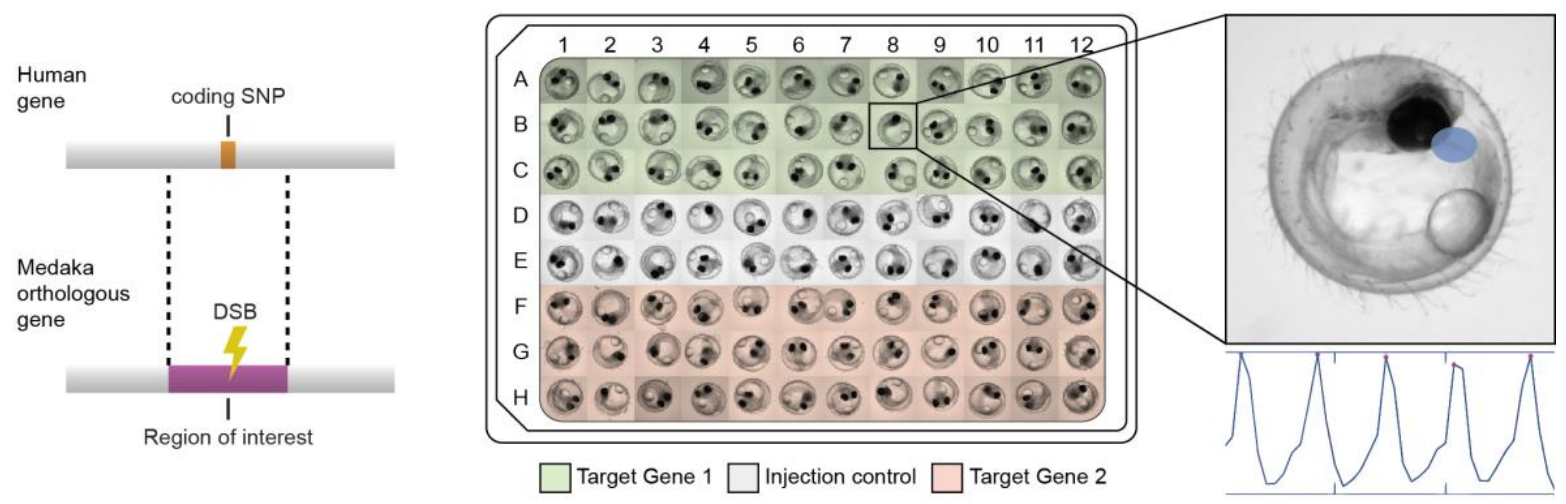

B
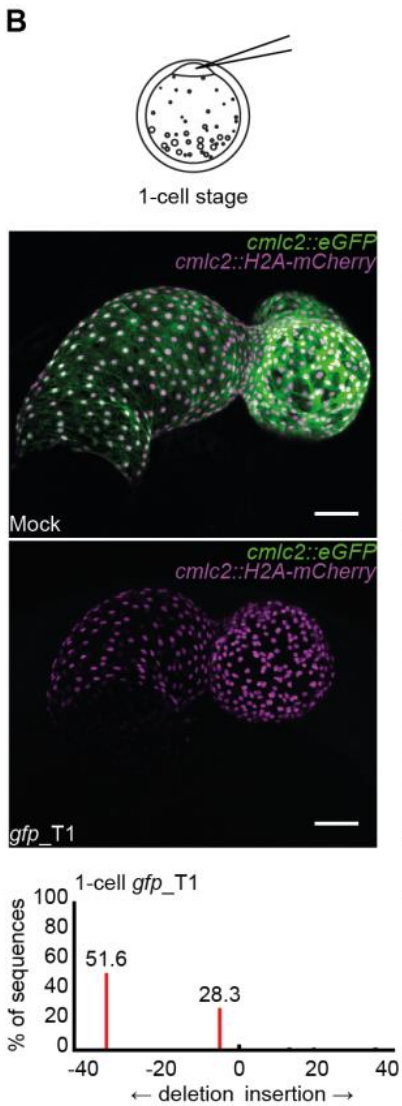

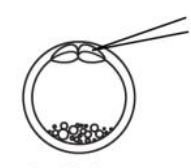

4-cell stage
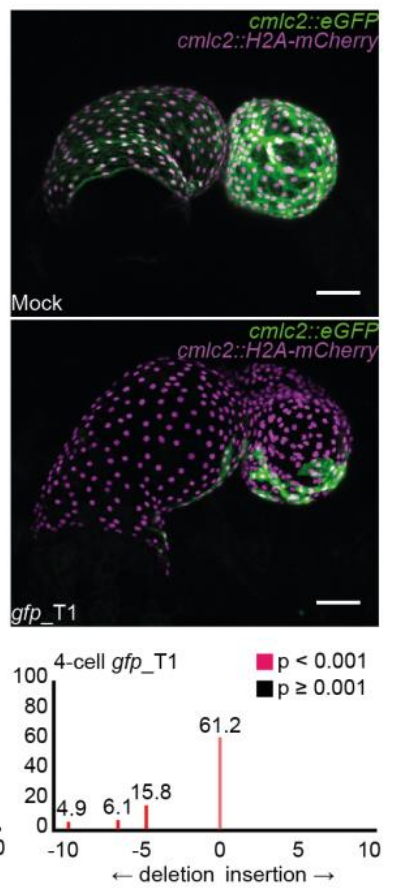

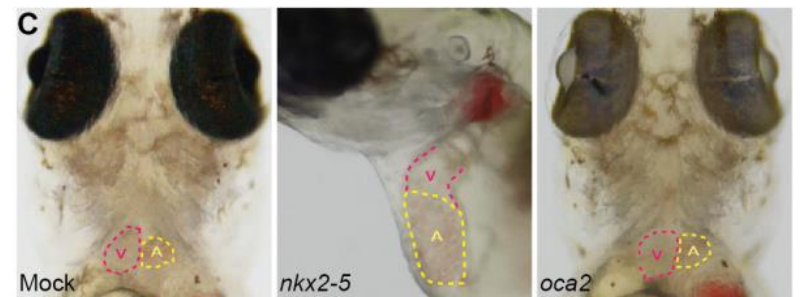

D
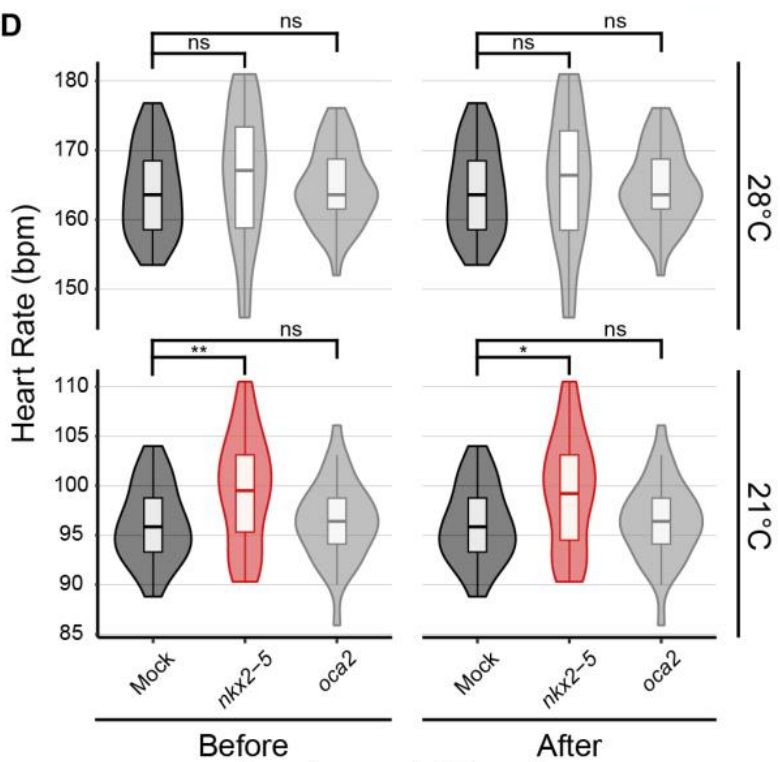

Developmental Focusing

Figure 1. Functional gene validation pipeline confirms heart rate phenotype in $n k x 2-5$ crispants A Schematic overview of our functional gene validation pipeline: position of human coding SNP mapped to medaka orthologous gene to define region of interest for CRISPR/Cas9 gene targeting (double strand break; DSB). 96-well plate layout of crispant embryos (Target Gene 1 and 2) separated by GFP mRNA mock-injected siblings. Embryos are subjected to high-throughput imaging followed by automated heart detection (blue area) and heart rate quantification (graphical output; HeartBeat software (Gierten et al. 2020). B Confocal images of GFP expression in mock-injected and $g f p$ crispant embryo hearts of the $c m l c 2:: e G F P c m l c 2:: H 2 A-m C h e r r y$ reporter line $(7 \mathrm{dpf})$. Embryos were injected either at the 1-cell or 4-cell stage. Note: complete loss of GFP expression when injected at the 1-cell stage $(\mathrm{n}=$ $8 / 8)$, while mosaic expression when injected at 4 -cell stage $(n=4 / 4)$. Genotyping of $g f p$ crispants display 
98

the genetic mosaicism resulting from Cas9-based targeting. Scale bars: $50 \mu \mathrm{m}$. For full image refer to Fig S1B. C Comparison of the atrium (A, dotted red line) and ventricle (V, dotted yellow line) in GFPinjected (Mock) and $n k x 2-5$ and oca 2 crispant embryos (9 dpf). Note: $n k x 2-5$ crispant shows dilated heart chambers while mock injected and oca 2 crispant embryos are indistinguishable. Loss of eye pigmentation in oca 2 crispants reflects high efficiency of knock-out rate in the injected generation. D Heart rate measurements (beats per minute, bpm) of GFP-injected (Mock; dark grey), $n k x 2-5$ and oca2 crispant embryos (4 dpf) at 21 and $28^{\circ} \mathrm{C}$, before (left) and after (right) exclusion of severely affected embryos (developmental focusing) reveal elevation of mean heart rates in $n k x 2-5$ targeted embryos, significant at $21^{\circ} \mathrm{C}$ (red). Significance was determined by two-tailed Student's t-test; *p< 0.05 , ** $p<$ 0.01 , ns (not significant; light grey). For biological replicates see Source Data Fig 1D.

function was fully developed and the heart rate had reached a plateau at 4 days post fertilization (Gierten et al. 2020) (4 dpf; developmental stage 31-32 (Iwamatsu 2004)).

To assess changes in mean heart rate with statistical significance, we took advantage of a 96well plate format, and imaged multiple biological replicates of crispants ( 3 rows; $n=36$ per condition) as well as of GFP $m R N A$ mock-injected siblings as internal plate control (2 rows; $\mathrm{n}=24$ ) (Fig S2A). To assess heart function under different environmental conditions, embryos were imaged at two different temperatures $\left(21\right.$ and $28^{\circ} \mathrm{C}$, respectively). Heart rates of all embryos were quantitatively determined from the imaging data using the HeartBeat software (Gierten et al. 2020), and randomly selected embryos were genotyped to correlate CRISPR/Cas9 targeting (Hammouda et al. 2019).

While mock injected embryos did not show phenotypes, crispants of the positive control $n k x 2$ 5 displayed a variety thereof. These ranged from global severe developmental delays to local cardiac malformations morphologically resembling the phenotypes previously observed in zebrafish $n k x 2-5$ mutants such as enlarged heart chambers (Fig 1C) (Targoff et al. 2013). Notably, in the negative control (oca2 crispants) neither cardiac nor developmental phenotypes were observed (Fig 1C), indicating that genome targeting of oca2 as well as injection and handling of the embryos did not impact on heart and general development per se. Quantitative comparison of cardiac function revealed an overall elevation in the mean heart rate of $n k x 2-5$ crispants $\left(21^{\circ} \mathrm{C} 99.7 \mathrm{bpm}, 28^{\circ} \mathrm{C} 166 \mathrm{bpm}\right)$ compared to mock control siblings $\left(21^{\circ} \mathrm{C} 96.1 \mathrm{bpm}\right.$, $28^{\circ} \mathrm{C} 164 \mathrm{bpm}$ ) with a significant $(\mathrm{p}=0.0074)$ difference at $21^{\circ} \mathrm{C}$ (Fig 1D; left panel). Notably, independent experimental replicates targeting the same $n k x 2-5$ exon with two different sgRNAs robustly yielded a significant heart rate phenotype at $21^{\circ} \mathrm{C}$ (Fig S2B-C). In contrast, the mean heart rate in oca 2 crispants was indifferent from mock control at either temperature $\left(21^{\circ} \mathrm{C} 96.4\right.$ bpm, $\left.28^{\circ} \mathrm{C} 165 \mathrm{bpm}\right)$, validating $o c a 2$ as bona fide negative control.

6 
123 To avoid severe developmental delays in $n k x 2-5$ crispants to potentially skew heart rate 124 comparisons, we further applied a developmental focusing filter. Only embryos having 125 developed beyond stage 28 (Iwamatsu 2004), at which cardiac function was previously shown 126 to have reached a functional plateau (Gierten et al. 2020), were chosen for statistical analysis.

127 Developmental focusing, only excluded three embryos from the $n k x 2-5$ group which did not 128 impact on the results (Fig 1D; right panel). These results underline the robustness of our 129 pipeline and demonstrate its sensitivity to detect mild heart rate phenotypes reflecting cardiac 130 function already at embryonic stages of medaka development.

132 Next, we determined the baseline probability of heart rate phenotypes by targeting a set of 133 randomly selected genes with CRISPR/Cas9. From a total of 23622 annotated medaka coding 134 genes in Ensembl (Yates et al. 2020), we used a random number generator to select 10 genes 135 (Table 1). For each gene, a random exon was chosen for targeting via CRISPR/Cas9. Heart 136 rates of 36 crispants were assessed per locus. To control for potential heart rate fluctuations in 137 embryos within and across different experiments (i.e. 96-well plates), we included mock138 injected siblings as internal plate control. Heart rates of target gene crispants and control siblings were scored and the means were compared before and after developmental focusing.

\section{Table 1. List of randomly selected genes.}

142 Medaka Ensembl gene names and codes, as well as orthologous human genes as annotated in the $95^{\text {th }}$ 143 Ensembl release.

\begin{tabular}{|l|l|l|}
\hline Ensembl ID & Medaka Gene & Orthologous Human Gene \\
\hline ENSORLG00000006335 & novel gene - cdc42 & na (CDC42 by name) \\
\hline ENSORLG00000000979 & novel gene - ogdh & OGDH \\
\hline ENSORLG00000005268 & duox & DUOX1 \\
\hline ENSORLG00000007310 & git2 & GIT2 \\
\hline ENSORLG00000003492 & mus81 & MUS81 \\
\hline ENSORLG00000020766 & orl24-2 & na \\
\hline ENSORLG00000007400 & plekha8 & PLEKHA8 \\
\hline ENSORLG00000022757 & ttl & TTL \\
\hline ENSORLG00000005922 & cabp4 & CABP2 \\
\hline ENSORLG00000023106 & novel gene - eml6 & EML6 \\
\hline
\end{tabular}


145 Comparative heart rate analysis revealed a heart rate phenotype in two out of the ten randomly 146 selected genes at both temperatures measured (Fig 2, and Fig S3). Remarkably, both genes of 147 the random set, the oxoglutarate dehydrogenase (ogdh) and the cell division control protein 42 148 homolog ( $c d c 42)$ have been previously associated with heart phenotypes in human GWAS or 149 were shown to play a role in heart function, respectively (Thanassoulis et al. 2013; Qian et al.

150 2011; Li et al. 2017). These results confirm the reliability of our assay to identify genes of a 151 given set that affect cardiac function. Of note, repeated rounds of random gene selection 152 revealed a similar mean baseline of $10-20 \%$ of selected genes being related to the heart in 153 GWAS or previous experimental reports.

154

A

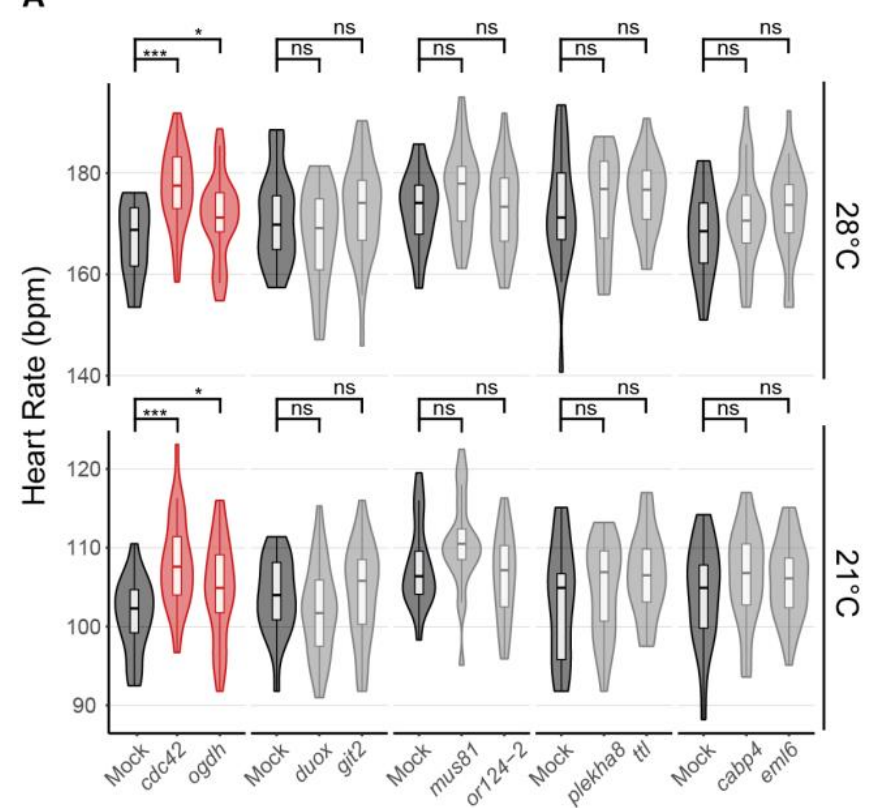

B

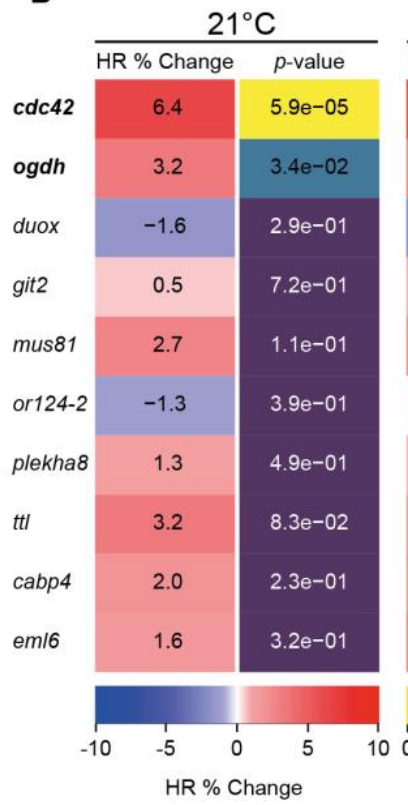

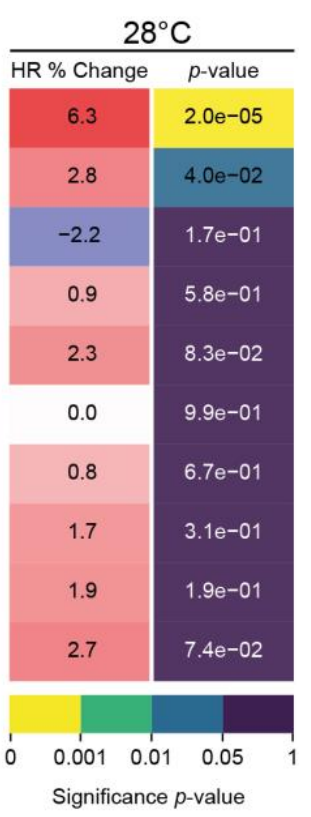

Figure 2. Baseline probability of heart rate phenotype assessed via in vivo targeting of randomly selected genes

A Heart rate measurements (beats per minute, bpm) of GFP-injected (Mock) and corresponding sibling crispant embryos ( $4 \mathrm{dpf})$ at 21 and $28^{\circ} \mathrm{C}$ after developmental focusing. Different experimental plates are represented by breaks on the x-axis. Significant differences in mean heart rates were determined between each crispant embryo group and its corresponding sibling control group by two-tailed Student's t-test; ${ }^{*} p<0.05, * * * p<0.001$, ns (not significant). Crispants showing significant heart rate phenotype (red), GFP-injected controls (Mock; dark grey), crispants showing no significant heart rate phenotype (light grey). B Heatmap quantitative representation of the data shown in (A); for each measured temperature, the percent change in mean heart rate (HR \% Change) between crispants and their corresponding control sibling, flanked by the statistical significance ( $p$-value) of the observed change calculated by two-tailed Student's t-test on the full distribution in (A). Genes showing significant heart rate phenotypes are indicated in bold. For biological replicates see Source Data Fig S3. 
156 We next applied our pipeline to interrogate a larger, targeted selection of genes associated with

157 cardiovascular diseases in human GWAS. We used GRASP (Eicher et al. 2015), the genome158 wide repository of associations between single nucleotide polymorphisms (SNPs) and 159 phenotypes, to compile a list of 40 candidate genes from human GWAS with a coding 160 association to heart phenotypes (hGWAS genes; Table 2). We focused on genes with no prior 161 experimental link to heart function, while including few known heart genes as additional 162 positive controls. To address the specificity of our approach, the selected candidate genes were 163 categorized according to their association into general heart related $(n=17)$ or related specifically to heart rate $(n=23$; Table2).

166 Heart rates of candidate gene crispants and control injected siblings were scored and compared 167 before (Fig S5) and after developmental focusing (Fig 3A and Figure S4). Across the hGWAS 168 set of 40 genes, comparative heart rate analysis showed statistically significant heart rate 169 phenotypes in a total of 16 genes (Fig 3B). The five positive controls, known to play key roles 170 in heart functions such as cardiac contraction (TTN (Itoh-Satoh et al. 2002) and NACA (Park et 171 al. 2010)) and heart rate regulation (CASQ2 (Faggioni \& Knollmann 2012), KCNH2 (Gianulis 172 \& Trudeau 2011) and SCN5A (Zaklyazminskaya \& Dzemeshkevich 2016; Huttner et al. 2013)) 173 clearly responded in the assay. Beyond known cardiac genes, we revealed new genes linked to 174 various biological functions (CCDC141, GIGYF1, HOMEZ, MYRF, SMG6, CMYA5, CNOT1, 175 SLC17A3, TRAPPC12, SSPO and PADI4) which up to now, had little to no experimental 176 evidence in cardiac function (Rathjens et al. 2020; Benson et al. 2017; Yamaguchi et al. 2018; 177 Elmen et al. 2020).

178 When analyzing the candidates according to their GWAS association ("heart rate" and "non179 heart rate" phenotypes), we observed a strong positive correlation between the respective 180 phenotypes observed in medaka crispants already at the embryonic stages used and the 181 associated phenotype in adult human GWAS. The proportion of heart rate-associated genes in 182 hGWAS that yield a heart rate phenotype in medaka embryos (13/23) was elevated compared 183 to the proportion of non-heart rate-associated genes yielding a heart rate phenotype (3/17). Even 184 when considering the entire group, we observed a higher proportion of genes with an effect on 185 heart rate in our targeted hGWAS gene set (16/40) compared to our randomly selected gene set 186 (2/10) (Fig 3C). Taken together, phenotypes in early medaka embryos likely reflect risk factors 9 
Table 2. List of candidate genes extracted from Human GWAS using GRASP 2.0 Database

Human genes are categorized according to their association into "heart rate" (bold) and "non-heart rate" (non-bold) related phenotypes in human GWAS.

\begin{tabular}{|l|l|l|l|l|l|}
\hline $\begin{array}{l}\text { Human } \\
\text { Gene }\end{array}$ & $\begin{array}{l}\text { Coding SNP } \\
\text { ID }\end{array}$ & $\begin{array}{l}\text { Associated heart } \\
\text { phenotype }\end{array}$ & $\begin{array}{l}\text { Association } \\
\text { Reference }\end{array}$ & $\begin{array}{l}\text { Medaka orthologue gene (Ensembl } \\
\text { release) }\end{array}$ & Ensembl Gene Code \\
\hline ATP8B4 & rs2452524 & Pulse rate & (Hiura et al. 2010) & atp8b4 (98) & ENSORLG00000005106 \\
\hline CASQ2 & rs4074536 & QRS interval & $\begin{array}{l}\text { (Sotoodehnia et al. } \\
\text { 2010) }\end{array}$ & casq2 (89) & ENSORLG00000017885 \\
\hline CCDC141 & rs17362588 & Heart rate & (Hoed et al. 2013) & na (89) (TBLASTN=ccdc141) & TBLASTN = ENSORLG00000030409 \\
\hline CEP85L & rs3734381 & QRS interval & $\begin{array}{l}\text { (Sotoodehnia et al. } \\
\text { 2010) }\end{array}$ & cep85l (91) & ENSORLG00000015455 \\
\hline CMYA5 & rs10942901 & Heart rate & (Hoed et al. 2013) & cmya5 (91) & ENSORLG00000008983 \\
\hline COL9A1 & rs592121 & Pulse rate & (Hiura et al. 2010) & col9a1b (98) & ENSORLG00000010431 \\
\hline GIGYF1 & rs221794 & Heart rate & (Hoed et al. 2013) & gigyf1 (89) & ENSORLG00000003655 \\
\hline GRID2 & rs1385405 & Pulse rate & (Hiura et al. 2010) & grid2 (98) & ENSORLG00000024663 \\
\hline HOMEZ & rs1055061 & Sick sinus syndrome & (Holm et al. 2011) & homeza (89) & ENSORLG00000012220 \\
\hline KCNH2 & rs1805123 & QT interval & (Pfeufer et al. 2009) & kcnh2 (98) & ENSORLG00000004137 \\
\hline MINAR1 & rs2297773 & Pulse rate & (Hiura et al. 2010) & minar1 (98) & ENSORLG00000016707 \\
\hline
\end{tabular}

Hammouda et al., 2021 Validating understudied GWAS heart genes 


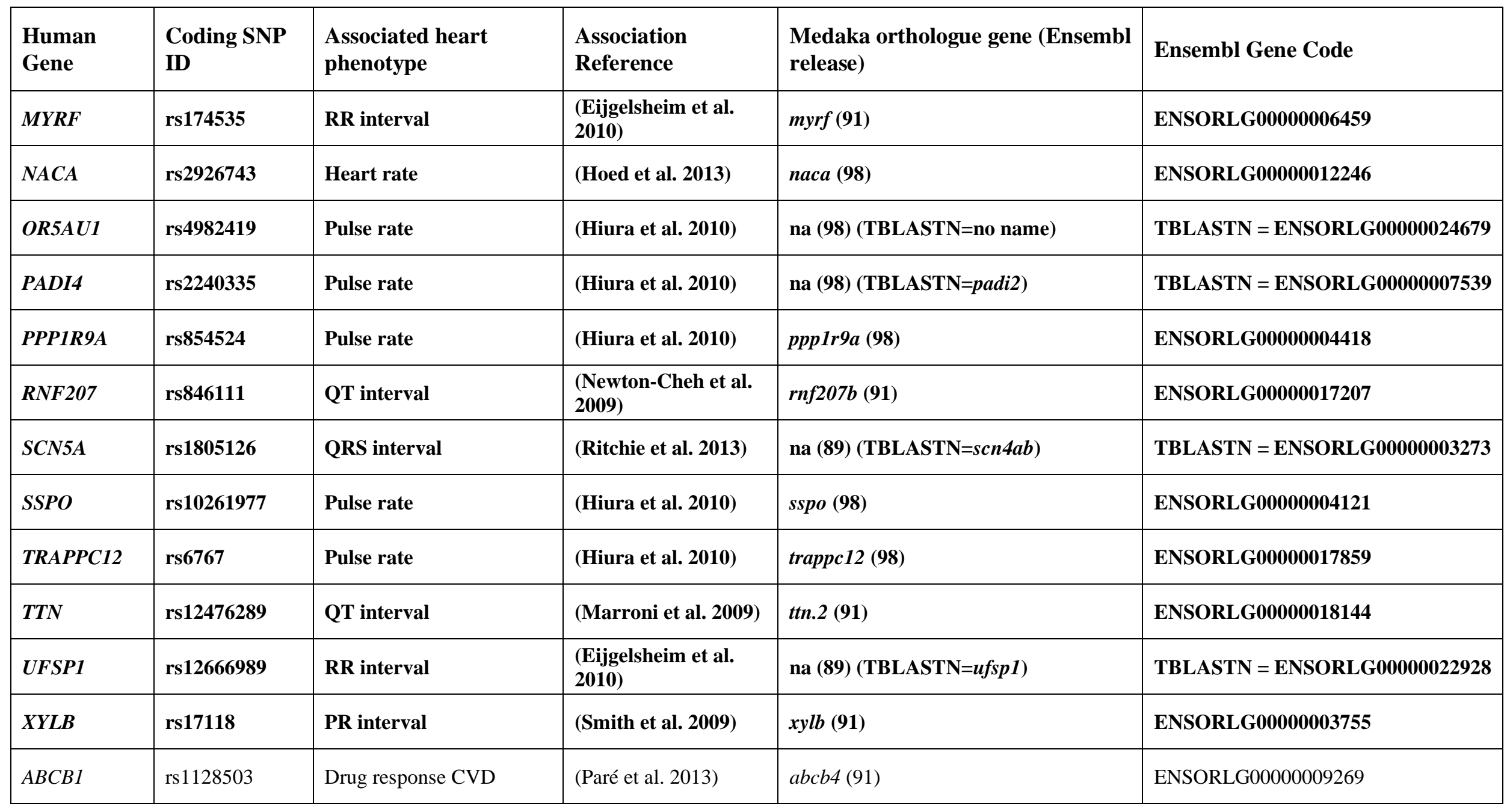

11

Hammouda et al., 2021 Validating understudied GWAS heart genes 


\begin{tabular}{|c|c|c|c|c|c|}
\hline $\begin{array}{l}\text { Human } \\
\text { Gene }\end{array}$ & $\begin{array}{l}\text { Coding SNP } \\
\text { ID }\end{array}$ & $\begin{array}{l}\text { Associated heart } \\
\text { phenotype }\end{array}$ & $\begin{array}{l}\text { Association } \\
\text { Reference }\end{array}$ & $\begin{array}{l}\text { Medaka orthologue gene (Ensembl } \\
\text { release) }\end{array}$ & Ensembl Gene Code \\
\hline$B A G 3$ & rs3858340 & $\begin{array}{l}\text { Sporadic dilated } \\
\text { cardiomyopathy }\end{array}$ & (Villard et al. 2011) & bag3 (89) & ENSORLG00000013813 \\
\hline CLCNKA & rs 1805152 & $\begin{array}{l}\text { Sporadic dilated } \\
\text { cardiomyopathy }\end{array}$ & (Villard et al. 2011) & clcnk (89) & ENSORLG00000018693 \\
\hline CNOT1 & rs11866002 & Aortic valve calcium & $\begin{array}{l}\text { (Thanassoulis et al. } \\
\text { 2013) }\end{array}$ & cnotl (91) & ENSORLG00000013734 \\
\hline EDN1 & rs 150035515 & Aortic valve calcium & (Matsa et al. 2014) & edn1 (89) & ENSORLG00000009276 \\
\hline $\mathrm{HCN} 4$ & rs529004 & Aortic valve calcium & $\begin{array}{l}\text { (Thanassoulis et al. } \\
\text { 2013) }\end{array}$ & hcn4 (91) & ENSORLG00000013180 \\
\hline$M A M L 3$ & rs11729794 & $\begin{array}{l}\text { Congenital heart } \\
\text { malformations }\end{array}$ & (Hu et al. 2013) & na (91) $($ TBLASTN=maml3) & $\begin{array}{l}\text { TBLASTN = NCBI Ref Seq: } \\
\text { XM_023954746.1 }\end{array}$ \\
\hline$N U B P 2$ & rs344359 & LV systolic dysfunction & (Vasan et al. 2009) & nubp2 (91) & ENSORLG00000007228 \\
\hline PIEZO1 & rs2290902 & Bicuspid aortic valve & (Wooten et al. 2010) & piezol $(91)$ & ENSORLG00000000402 \\
\hline$P L G$ & rs 13231 & Aortic valve calcium & $\begin{array}{l}\text { (Thanassoulis et al. } \\
\text { 2013) }\end{array}$ & plg (91) & ENSORLG00000020532 \\
\hline$R G S 3$ & rs 12341266 & $\begin{array}{l}\text { Hypertrophic } \\
\text { cardiomyopathy }\end{array}$ & (Wooten et al. 2013) & $\operatorname{rgs} 3 a(91)$ & ENSORLG00000006823 \\
\hline SCMH1 & rs 10489520 & Ischemic stroke & (Ikram et al. 2009) & scmhl (91) & ENSORLG00000014207 \\
\hline$S H 2 B 3$ & rs3184504 & Tetrology of fallot & (Cordell et al. 2013) & $\operatorname{sh} 2 b 3(91)$ & ENSORLG00000003569 \\
\hline SLC17A3 & rs942379 & Bicuspid aortic valve & (Wooten et al. 2010) & si:ch1073-513e17.1 (91) & ENSORLG00000007671 \\
\hline
\end{tabular}

12

Hammouda et al., 2021 Validating understudied GWAS heart genes 


\begin{tabular}{|l|l|l|l|l|l|}
\hline $\begin{array}{l}\text { Human } \\
\text { Gene }\end{array}$ & $\begin{array}{l}\text { Coding SNP } \\
\text { ID }\end{array}$ & $\begin{array}{l}\text { Associated heart } \\
\text { phenotype }\end{array}$ & $\begin{array}{l}\text { Association } \\
\text { Reference }\end{array}$ & $\begin{array}{l}\text { Medaka orthologue gene (Ensembl } \\
\text { release) }\end{array}$ & Ensembl Gene Code \\
\hline SMG6 & rs216193 & Aortic root size & (Vasan et al. 2009) & smg6 (91) & ENSORLG00000003317 \\
\hline VEPH1 & rs1378796 & $\begin{array}{l}\text { Sporadic dilated } \\
\text { cardiomyopathy }\end{array}$ & (Villard et al. 2011) & veph1 (89) & ENSORLG00000012452 \\
\hline ZFHX3 & rs2228200 & Aortic valve calcium & $\begin{array}{l}\text { (Thanassoulis et al. } \\
\text { 2013) }\end{array}$ & zfhx3 (91) & ENSORLG00000007874 \\
\hline
\end{tabular}

190

Hammouda et al., 2021 Validating understudied GWAS heart genes 
bioRxiv preprint doi: https://doi.org/10.1101/2021.02.03.429563; this version posted June 21,2021 . The copyright holder for this preprint (which was not certified by peer review) is the author/funder, who has granted bioRxiv a license to display the preprint in perpetuity. It is made available under aCC-BY-NC-ND 4.0 International license.

A

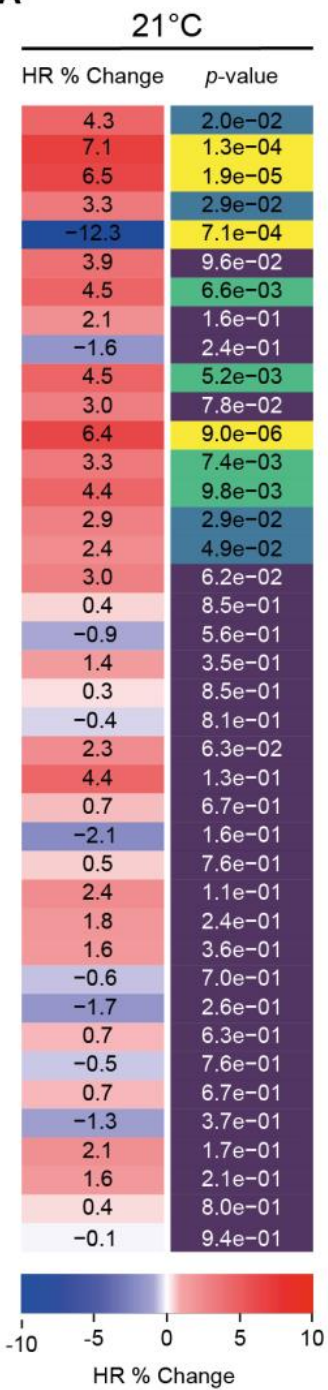

B

Genes with significant HR phenotypes

$21^{\circ} \mathrm{C}$ only $28^{\circ} \mathrm{C}$ only

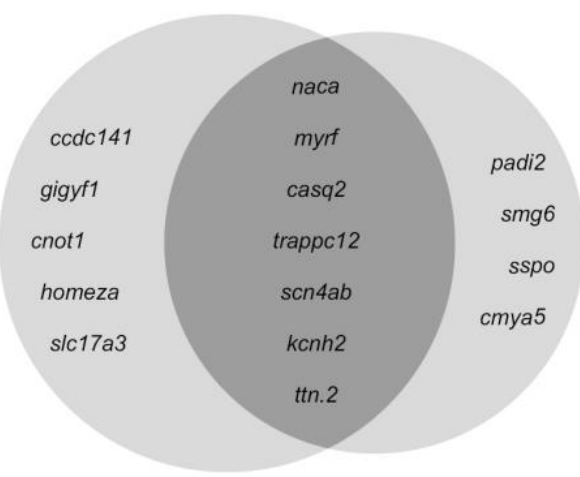

C

Proportions of genes with HR phenotype

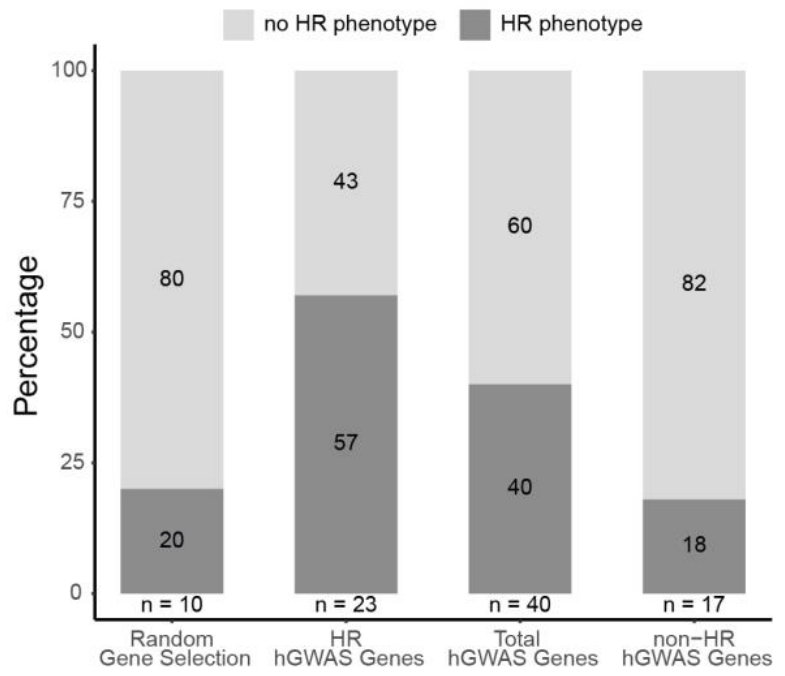

Figure 3 Targeted human heart-GWAS validations reveal new genes affecting heart rate

A Heatmap quantitative representation of the comparative heart rate analysis between each crispant embryo group and its corresponding control sibling group after developmental focusing (also see plots in Fig S4); for each measured temperature, the percent change in mean heart rate (HR \% Change) between crispants and their corresponding control sibling, flanked by the statistical significance ( $p$ value) of the observed change calculated by two-tailed Student's t-test on the full distribution (Fig S4). Genes showing significant heart rate phenotypes are indicated in bold. For biological replicates see Source Data Fig S4. B Venn diagram summarizing the genes with significantly different heart rate (HR) phenotypes only at $21^{\circ} \mathrm{C}$, only at $28^{\circ} \mathrm{C}$ or at both temperatures (dark grey). C Stacked plots representing percentage of genes showing a significant heart rate phenotype (dark grey) in each group. Number of genes for each group is denoted $(n)$. hGWAS corresponds to the selection of genes associated to heart phenotypes in human GWAS.

193 in human adults, thus we uncovered functionally relevant heart rate phenotypes in previously

194 uncharacterized genes. 
195 In addition to the observed heart rate phenotypes in trappc12 crispants, we also uncovered

196 morphological heart phenotypes such as heart looping defects. Where in wild-type medaka,

197 heart looping usually starts at around stage 27, when the atrium shifts to the right and lies

198 adjacent to the ventricle (Iwamatsu 2004), trappc12 crispants revealed strong heart looping

199 phenotypes (12/46) not observed in oca2 crispants (0/22) or mock-injected embryos (0/26) (Fig 200 4A).

201 In crispants of $s c n 4 a b$, heart rate analysis interestingly uncovered a bimodal distribution, with 202 a population displaying roughly half the average heart rate at both recorded temperatures (Fig 203 4B). Visual inspection of the $s c n 4 a b$ crispant embryos revealed an arrhythmic heart anomaly 204 previously reported in zebrafish scn5a mutants (Huttner et al. 2013), i.e. known as an atrio205 ventricular block (AV-block), characterized by a delay or disruption of impulse transmission 206 from the atrium to the ventricle. Scoring the beat frequency of both heart chambers separately 207 in individual embryos exposed the impaired rhythm of atrial to ventricular contractions, which 208 resulted in a delay or even skipping of ventricular beats in the scn $4 a b$ crispants but not in control 209 siblings (Fig 4C). scn4ab crispants displayed various severities of the AV-block from mild 210 (regular heart beats with occasional beat skipping), to moderate (consistent 2:1 atrial to 211 ventricular contraction; Movie S1), to severe (3:1 or more; Movie S2). Still heavily affected $212 s c n 4 a b$ crispants survived until hatching. Impressively, the prevalence of the arrhythmia 213 phenotype in scn $4 a b$ crispants was markedly high, exceeding $90 \%$ of the injected embryos, 214 reflecting the high efficiency of the heiCas 9 and the high penetrance of the mutations 215 introduced. Notably, the arrhythmia phenotype of scn4ab mutants bred to homozygosity did 216 not differ from the phenotype observed in the injected generation F0 (Movie S1), verifying the 217 specificity of the phenotype. These results further underscore the efficacy and reliability of 218 medaka F0 crispant analysis as a rapid validation tool to identify genes with a functional link 219 to human cardiac diseases.

\section{Discussion}

Most cardiovascular diseases can be prevented if diagnosed and treated early. Previous studies

224 have shown the importance of the resting heart rate as a vital risk factor both in terms of 225 prediction and prevention of CVDs (DYER et al. 1980; MD et al. 2010; Eppinga et al. 2016). 


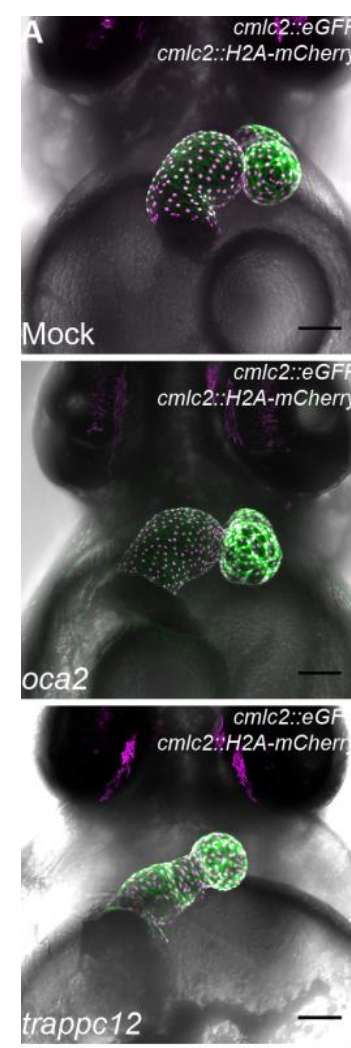

B

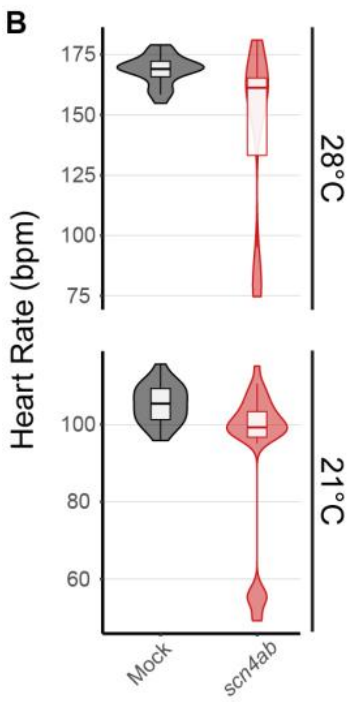

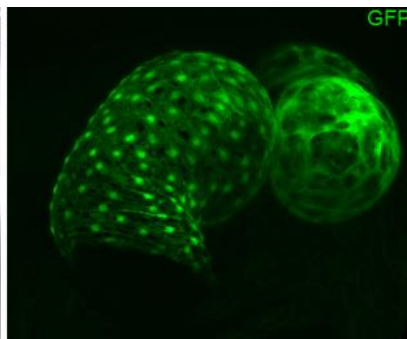
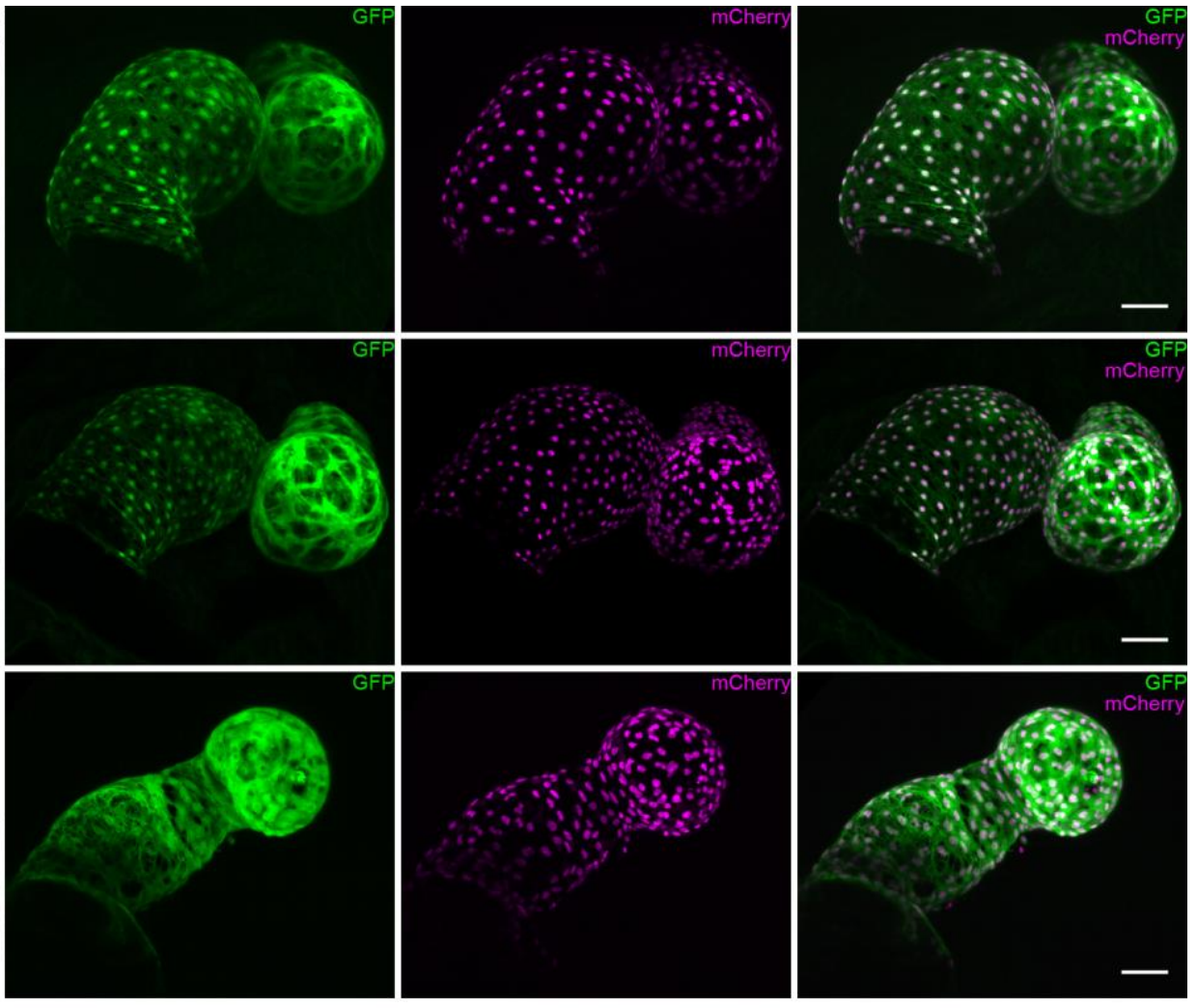

C

$21^{\circ} \mathrm{C}$

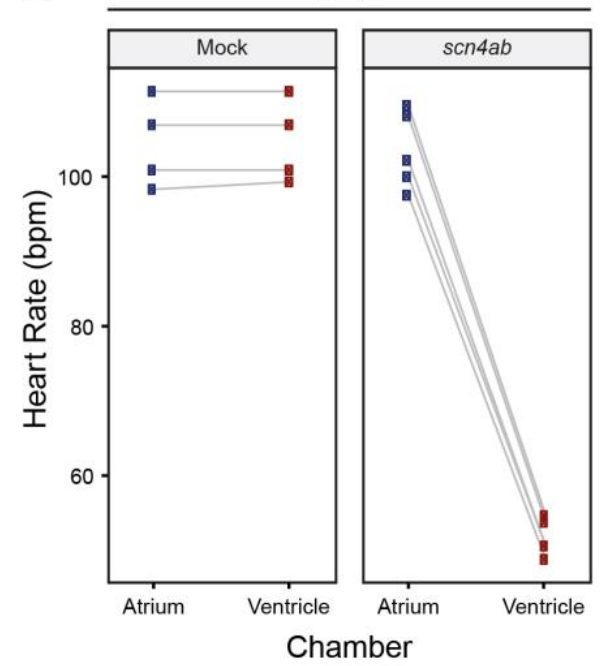

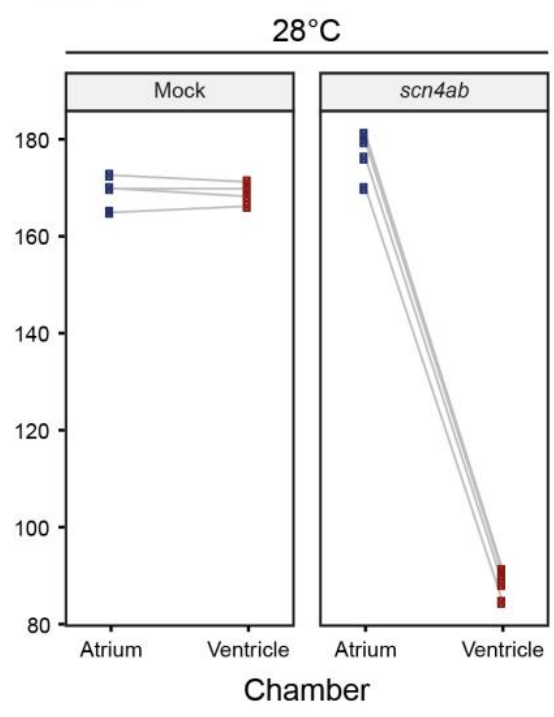

Figure 4. Heart looping defects and AV-block type arrhythmia in medaka crispant embryos

A Confocal images of hearts of mock-injected, oca2 and trappc12 crispants of the cmlc2::eGFP cmlc2::H2A-mCherry reporter line (7 dpf); note the heart looping defect observed in trappc12 crispants. Scale bars: $100 \mu \mathrm{m}$ (First panel on left) and $50 \mu \mathrm{m}$ (blow-up images). B Heart rate measurements (beats per minute, bpm) of GFP-injected (Mock; dark grey; $\mathrm{n}=22$ ) and $s c n 4 a b$ crispant (red; $\mathrm{n}=32$ ) embryos ( $4 \mathrm{dpf}$ ) at 21 and $28^{\circ} \mathrm{C}$; note the bimodal distribution in $s c n 4 a b$ crispants. $\mathrm{C}$ Paired plots showing heart rate scores for each chamber separately (atrium in blue; ventricle in red) of individual embryos from $\mathrm{B}$ at both temperatures. 
An increase of 5 beats per minute correlates with a $20 \%$ increase in risk of mortality (Eppinga et al. 2016), and reducing the resting heart rate has proven to improve the clinical outcomes of various CVDs (Beere et al. 1984; MD et al. 2010).

230 Human GWAS have been performed in search of genetic determinants of CVDs, and although 231 a wide array of candidate genes with various functions are being associated to heart phenotypes 232 in human GWAS, further focus is usually turned to those few genes with pre-existing indication 233 of cardiac function. Other associated genes with an unknown function or without a pre-existing 234 functional link to the heart are often neglected and not pursued further, pushing all those into a 235 blind spot, resulting in a negative loop of discovery. Thus, it is important to address the role of such genes in heart function through experimental validation in model organisms, in pursuit of novel markers for CVD diagnosis. We address this blind spot of discovery by applying a highthroughput heart rate imaging and analysis pipeline coupled to a reverse genetic validation approach via CRISPR/Cas9 mediated mutagenesis in a genetically suited vertebrate model (Fig 240 1A).

241 F0 mutagenesis screens are becoming more and more popular (Teboul 2017; Shankaran et al. 2018; Wu et al. 2018; Heyde et al. 2020), largely due to the improvements in CRISPR/Cas9 gene targeting efficiency by modification of the enzyme or promoting nuclear localization 244 (Thumberger et al. 2021). Overall, gene targeting in medaka using CRISPR/Cas9 has proven to be highly efficient, as shown by the prominent loss of GFP expression in $g f p$ crispants when injected at the 1-cell stage (Fig 1B and Fig S1) and the prominent bi-allelic mutagenesis as apparent by the loss of eye-pigmentation in the oca2 crispants (Fig 1C and Fig S2A). A similarly high penetrance was also observed in the scn $4 a b$ crispants, where we detected and quantified severe arrhythmia phenotypes such as AV-block with our assay (Fig 4B-C, Movies S1 and S2). A $90 \%$ prevalence of the arrhythmia phenotype and an absence of global phenotypes further reflect the specificity of this phenotype.

252 A subset of the target genes in our assay (e.g. $n k x 2-5$, smg6, naca, ttn.2, abcb4) however, 253 yielded a rather broad range of global developmental phenotypes, potentially reflecting their 254 important roles in embryonic development. To address this un-avoidable outcome when 255 tackling genes with broader function (e.g. transcription factors or essential genes), we applied 256 a developmental focusing filter in the analysis phase. Doing so, we avoid a biased assessment 257 of the heart rate by ensuring the comparability of the crispant embryos on a global 17 
developmental scale, which in turn allows emphasizing cardiac-specific effects. Interestingly however, developmental focusing, although deemed important, in only few cases significantly altered the outcome of the analysis (Fig. S5). This reflects the robustness of the assay and the homogeneity of CRISPR/Cas9-induced phenotypes in the isogenic background of the medaka

262 line used.

263 In a set of ten randomly chosen genes we observed a baseline occurrence of heart-affecting genes of about $20 \%$ (Fig 2, and Fig S3). Relevantly, both genes were implicated in heart functions, further reflecting the reliability of our model and approach. For $c d c 42$, there is $a$ priori evidence of its human orthologue in heart development as well as in regulating heart function across species (Qian et al. 2011; Li et al. 2017). Surprisingly, we did not find any associations (coding or non-coding) of CDC42 to heart phenotypes in human GWAS according to the GRASP database (Eicher et al. 2015). As for $o g d h$, no experimental evidence in cardiac function has been previously reported, but a polymorphism located on one of its exons has been associated to heart phenotypes in human GWAS (Thanassoulis et al. 2013). Except for duox, which has been reported as having an indirect role in cardiac regeneration in zebrafish (Han et al. 2014), none of the other randomly selected genes were, to our knowledge, ever connected with cardiac function. In summary, from our random gene set, the only two affecting the heart beat ( $o g d h$ and $c d c 42$ ) are connected to cardiac function by prior evidence (Thanassoulis et al. 2013; Qian et al. 2011; Li et al. 2017). All but one positive ( $\mathrm{HCN} 4)$ control among the hGWAS candidates resulted in a pronounced heart rate phenotype in our assay, reflecting their role in cardiac contraction (TTN (Itoh-Satoh et al. 2002) and NACA (Park et al. 2010)), cardiac conduction and heart rate regulation (CASQ2 (Faggioni \& Knollmann 2012), KCNH2 (Gianulis \& Trudeau 2011) and SCN5A (Zaklyazminskaya \& Dzemeshkevich 2016; Huttner et al. 2013)). In case of the missing heart rate phenotypes anticipated in hcn 4 crispants, we suspect compensation by its paralog hcn $4 l$. For eleven hGWAS candidate genes, our analysis provided the first experimental evidence validating a cardiac function, and accordingly put these identified genes under the spotlight as new targets for future in-depth characterization and as candidates for the prediction of heart diseases prior to their onset. This is impressively substantiated by the emerging studies on the et al. 2020). 
Throughout our study, we primarily focused on the heart rate as a measure of cardiac function due to its ease of quantification and interpretation in high-throughput. However, we did also notice heart morphological phenotypes in some crispants across our hGWAS candidates list. Among others, trappc12 crispants showed heart looping defects, resulting in the improper placement of the atrium compared to the ventricle (Fig 4A). With advances in AI-based image analysis, important morphological features could be additionally extracted in high throughput, leading to a better understanding of factors involved in heart development and function. Grouping the candidate genes according to their heart GWAS association into heart rate and non-heart rate-related phenotypes further exposed the prominent positive correlation between the associated human phenotype and the observed phenotype in medaka (Fig 3C). Medaka's isogenic background, a product of inbreeding over multiple generations (Wittbrodt et al. 2002), enabled the detection of subtle changes in heart rate immediately in F0 crispants. This accelerated the analysis and avoided the necessity to analyze homozygous offspring in the second and third generation after CRISPR targeting.

303 It is noteworthy that despite the evolutionary distance from fish to humans the medaka phenotypes match the class of hGWAS effects. This is even more relevant since the roles of the genes in medaka were validated in embryos, suggesting that the validated marker genes have predictive power in humans. This deep functional conservation emphasizes the potential of our approach for the identification and validation of novel predictive genetic markers for cardiovascular diseases in humans. We have showcased a highly versatile, sensitive and robust high-throughput reverse genetic validation assay to address the pool of understudied, neglected putative candidates. In the future, the combination of genetic validation and drug screening in a single platform building on our assay will facilitate the simultaneous identification of novel genetic players and interacting small molecules with rescuing power.

\section{Materials and Methods}

\section{Ethics Statement}

317 All fish are maintained in closed stocks at Heidelberg University. Medaka (Oryzias latipes) 318 husbandry (permit number 35-9185.64/BH Wittbrodt, Regierungspräsidium Karlsruhe) was 319 performed according to local animal welfare standards (Tierschutzgesetz $§ 11$, Abs. 1, Nr. 1) in 19 
accordance with European Union animal welfare guidelines (Bert et al. 2016). The fish facility is under the supervision of the local representative of the animal welfare agency. The following medaka stocks and transgenic lines were used: wild-type Cabs and cmlc2::eGFP cmlc2::H2AmCherry transgenic HdrR-II strain. Medaka embryos were used at stages prior to stage 42. Medaka were raised and maintained as described previously (Köster et al. 1997).

\section{Generation of the transgenic cmlc2 dual-reporter line}

327 For dual-color cardiac imaging, cmlc2::eGFP and cmlc2::H2A-mCherry transgenic medaka 328 lines were generated in the wild-type HdrR-II background. A modified version of pDestTol2CG (http://tol2kit.genetics.utah.edu/index.php/PDestTol2CG) was used containing a cmlc2::eGFP reporter cassette. For the second, nuclear reporter, the eGFP was replaced by an H2A-mCherry insert. Both plasmids were each co-injected at $10 \mathrm{ng} / \mu \mathrm{l}$ with $10 \mathrm{ng} / \mu 1 \mathrm{Tol} 2$ transposase mRNA into HdrR-II one-cell stage embryos using the microinjection technique as previously described (Rembold et al. 2006) to generate separate reporter lines. A double transgenic line was derived from a cross of the $c m l c 2:: e G F P$ line to $c m l c 2:: H 2 A-m C h e r r y$ line and maintained for CRISPRCas9 injections.

\section{Candidate gene selection}

338 For the unbiased gene targeting, an online random number generator was used to generate 10 numbers between 1 and 23622, corresponding to the number of annotated medaka coding genes in Ensembl (Yates et al. 2020) (Table 1). The number of exons for each gene was counted and a random number was generated to select the exon for CRISPR/Cas9 targeting. For the targeted human heart-GWAS (hGWAS) gene selection, the genome-wide repository of associations between SNPs and phenotypes (GRASP v2.0) was used (Eicher et al. 2015). In the search field, "Heart" and "Heart rate" were chosen as the respective categories for all heart- and heart raterelated phenotypes associated in human GWAS, only coding SNPs (i.e. SNP functional class = exons) were searched for. List of resulting genes was extracted (Table 2), and candidate genes

347 for the functional validation assay were chosen. The focus was on uncharacterized genes, or 348 genes with no prior experimental link to heart function, yet some known heart genes were 349 included as proof of concept. For each hGWAS candidate gene, the corresponding medaka ortholog was extracted using Ensembl (Yates et al. 2020). For the few genes which did not have 20 
an annotated medaka ortholog, the human protein sequence was BLASTed using the "tblastn"

352 function of the NCBI BLAST (https://blast.ncbi.nlm.nih.gov/Blast.cgi) and Ensemble 353 (http://www.ensembl.org/Multi/Tools/Blast) online tools to obtain a target medaka locus. Using

354 Geneious 8.1.9 (https://www.geneious.com), regions of interest (ROI) on medaka orthologous 355 genes for CRISPR targeting were primarily chosen based on the corresponding location of 356 human SNP when aligning the medaka and human protein sequences.

\section{$s g R N A$ target sites selection and in vitro transcription}

359 All sgRNA target sites used in this study are listed in Table S1. sgRNAs were designed with 360 CCTop as described in Stemmer et al. (Stemmer et al. 2015). sgRNA target sites were selected 361 based on number of potential off-target sites and their corresponding mismatches. Preferably, 362 sgRNAs selected had no off-target site or at least 3 nucleotide mismatches. sgRNA for oca2 363 was the same as in Lischik et al. (Lischik et al. 2019). Cloning of sgRNA templates and in vitro 364 transcription was performed as detailed in Stemmer, et al. (Stemmer et al. 2015). All sgRNAs 365 were initially tested after synthesis for in vivo targeting via injections into medaka embryos, 366 followed by genotyping using our filter-in-tips protocol (Hammouda et al. 2019), in brief terms, 367 by PCR amplification of target locus followed by T7 Endonuclease I assay (New England 368 Biolabs).

\section{Microinjection}

371 Medaka one-cell or four-cell stage embryos were injected into the cytoplasm as previously 372 described (Stemmer et al. 2015). Injection solutions for CRISPR targeting comprised: $150 \mathrm{ng} / \mu 1$ heiCas 9 mRNA (Thumberger et al. 2021), $15 \mathrm{ng} / \mu 1$ respective sgRNA and $10 \mathrm{ng} / \mu \mathrm{l}$ GFP mRNA as injection tracer. Control siblings were injected with $10 \mathrm{ng} / \mu \mathrm{l}$ GFP mRNA only. Injected embryos were incubated at $28^{\circ} \mathrm{C}$ in embryo rearing medium (ERM), screened for GFP expression at $1 \mathrm{dpf}$ and transferred to methylene blue-containing ERM (or plain ERM for reporter lines) and incubated at $28{ }^{\circ} \mathrm{C}$ until heart rate analysis (4 dpf) or confocal microscopy 378 (7 dpf). 


\section{Sample preparation and Imaging}

For the heart rate assay, one day prior to imaging ( $3 \mathrm{dpf})$, medaka embryos were transferred from methylene blue-containing ERM into plain ERM and incubated at $28{ }^{\circ} \mathrm{C}$. On day of imaging (4 dpf), individual medaka embryos (36 per sgRNA and 24 control injected) were administered to a $96 \mathrm{U}$-well microtiter plate (Nunc, Thermofisher \#268152) containing $200 \mu \mathrm{l}$ ERM per well and sealed using gas-permeable adhesive foil (4titude, Wotton, UK, 4ti0516/96). Plates were automatically imaged using an ACQUIFER Imaging Machine (DITABIS AG, Pforzheim, Germany) at 21 and $28^{\circ} \mathrm{C}$ with a 30-minute equilibration period before each measurement. Images were acquired in brightfield using $130 \mathrm{z}$-slices $(\mathrm{dz}=0 \mu \mathrm{m})$ and a $2 \mathrm{x}$ Plan UW N.A. 0.06 objective (Nikon, Düsseldorf, Germany) to capture the centered embryo. Integration times were fixed with $80 \%$ relative white LED intensity and 10 ms exposure time. Therefore, the whole 96-well plate was captured, with image sequences (videos) of entire microwells of approx. 10 seconds with 13 frames per second (fps). More details can be found in Gierten, et al. (Gierten et al. 2020).

For the live confocal microscopy of the reporter lines, injected embryos were treated from 4 dpf onwards with $5 x$ phenylthiourea (PTU) in ERM solution to prevent pigmentation. On the day of imaging (7 dpf), PTU solution was washed away with ERM, embryos were rolled on fine sand paper and de-chorionated by incubation in hatching enzyme. Following dechorionation, embryos were treated with $50 \mathrm{mM}$ 2,3-butanedione monoxime (BDM) in 1x Tricaine solution until de-coupling of heart beat ( $40 \mathrm{mins})$, which resulted in fully dilated heart chambers. Embryos were mounted on Matek dishes in 1.5\% low-melting agarose with $85 \mathrm{mM}$ BDM in 3x Tricaine solution. To avoid dehydration, mounted samples were covered with 30 $\mathrm{mM}$ BDM in 1x Tricaine solution throughout the imaging session. All confocal microscopy images were acquired at a Leica TCS SP8 with 10x dry or 20x oil objective, z-stacks of 200$300 \mu \mathrm{m}$ were acquired with a $\mathrm{z}$-step of $5 \mu \mathrm{m}$ or $1 \mu \mathrm{m}$ for $10 \mathrm{x}$ and $20 \mathrm{x}$ acquired images, respectively.

\section{HeartBeat detection and data analysis}

Image optimizations prior to analysis, as well as heart rate analysis using the HeartBeat software were performed as previously described (Gierten et al. 2020). In some instances, heart rates could not be scored due to inconvenient embryo orientations shielding the view of the 22 
heart. For scn $4 a b$ crispants with cardiac arrhythmias, atrium and ventricle for individual embryos were separately segmented, and the respective beating frequency for each chamber was measured. Data plots were generated using ggplot2 package (Wickham 2016) in R 3.6.1 (R Core Team, 2019) and R-studio 1.2.1335 (RStudio Team, 2018). Statistical analysis for heart rate comparisons were computed in R. Significant differences were determined by two-tailed Student's t-test. Significant $p$-values are indicated with asterisks $(*)$ with ${ }^{*} p<0.05, * * p<0.01$, $*_{* *} p<0.001$ and ns (not significant). Maximum intensity projections of confocal microscopy images were processed via Fiji image processing software.

\section{Embryo genotyping}

Nucleic acid extraction and genotyping of embryos was done as previously described (Hammouda et al. 2019). Briefly, after imaging, embryos in 96-well plate were lysed in $50 \mu 1$ Milli-Q water + $50 \mu \mathrm{l}$ Fin-Clip lysis buffer each (0.4 M Tris-HCl pH 8.0, 5 mM EDTA pH 8.0, $0.15 \mathrm{M} \mathrm{NaCl}, 0.1 \%$ SDS in Milli-Q water) using a custom 96-well mortar. The mortar was precleaned by incubation in hypochlorite solution (1:10 dilution of commercial bleach reagent) for at least 15 minutes followed by 5 minutes incubation in Milli-Q water. Plates containing lysed embryos were stored at $4{ }^{\circ} \mathrm{C}$ until genotyping. To confirm CRISPR on-target activity, per experimental plate, 2 embryos per condition were chosen at random for genotyping by PCR amplification of target locus using our filter-in-tips approach (Hammouda et al. 2019), followed by T7 Endonuclease I Assay (New England Biolabs). 30 PCR cycles were run in all samples, all primers used for PCR are listed in Table S2. Annealing temperatures were calculated using the online NEB Tm calculator (https://tmcalculator.neb.com/).

\section{Acknowledgments}

We thank A. Cornean for providing the $g f p_{-} \mathrm{T} 1$ guide RNA and for his help in acquiring the confocal images. We thank V. Weinhardt, E. Tsingos and all members of the Wittbrodt lab for their critical, constructive feedback on the procedure and the manuscript, F. Loosli and S. Lemke for their constructive feedback towards the project as well as J. Backs and E. Furlong for an outside perspective. We thank T. Kellner for excellent technical support. We acknowledge the excellent fish husbandry of E. Leist, M. Majewsky and A. Saraceno. We thank 23 
J. Gehrig (ACQUIFER Imaging GmbH) for supporting us with the Imaging machine. O.T.H. is and J.G. was a member of the Heidelberg Biosciences International Graduate School (HBIGS). This work was supported by a fellowship of the Deutsches Zentrum für HerzKreislauf-Forschung (DZHK) to O.T.H and through DFG CRC-1324 TP B4 and NIH 5R01ES029917 - 03, KiyosuTox. J.G. was supported by the Deutsche Herzstiftung e.V. (S/02/17) and by an Add-On Fellowship for Interdisciplinary Science of the Joachim Herz Stiftung.

\section{Author contributions}

O.T.H., T.T. and J.W. designed the study and implemented the methodology. J.G. generated the transgenic cmlc2 dual-reporter line. O.T.H., V.K. and M.Y.W. synthesized the guide RNAs. O.T.H. performed experiments. O.T.H. analyzed the data with contributions by M.Y.W. and T.T., and J.W. finalized the manuscript. J.W. provided resources and supervised this work.

\section{Conflict of interest}

All authors declare no competing interests.

\section{References}

465
Beere, P.A., Glagov, S. \& Zarins, C.K., 1984. Retarding effect of lowered heart rate on coronary atherosclerosis. Science, 226(4671), p.180.

Benson, M.A. et al., 2017. Ryanodine receptors are part of the myospryn complex in cardiac muscle. Scientific Reports, pp.1-12.

Bert, B. et al., 2016. Considerations for a European animal welfare standard to evaluate adverse phenotypes in teleost fish. The EMBO Journal, 35(11), pp.1151-1154.

Cambien, F. \& Tiret, L., 2007. Genetics of Cardiovascular Diseases. Circulation, 116(15), pp.1714-1724.

Cordell, H.J. et al., 2013. Genome-wide association study identifies loci on 12q24 and 13q32 associated with Tetralogy of Fallot. Human Molecular Genetics, 22(7), pp.1473-1481.

24 
DYER, A.R. et al., 1980. HEART RATE AS A PROGNOSTIC FACTOR FOR CORONARY HEART DISEASE AND MORTALITY: FINDINGS IN THREE CHICAGO EPIDEMIOLOGIC STUDIES. American Journal of Epidemiology, 112(6), pp.736-749.

Eicher, J.D. et al., 2015. GRASP v2.0: an update on the Genome-Wide Repository of Associations between SNPs and phenotypes. Nucleic Acids Research, 43(D1), pp.D799D804.

Eijgelsheim, M. et al., 2010. Genome-wide association analysis identifies multiple loci related to resting heart rate. Human Molecular Genetics, 19(19), pp.3885-3894.

Elmen, L. et al., 2020. Silencing of CCR4-NOT complex subunits affect heart structure and function. Disease Models \& Mechanisms, pp.dmm.044727-33.

Eppinga, R.N. et al., 2016. Identification of genomic loci associated with resting heart rate and shared genetic predictors with all-cause mortality. Nature Genetics, 48(12), pp.15571563 .

Faggioni, M. \& Knollmann, B.C., 2012. Calsequestrin 2 and arrhythmias. American Journal of Physiology-Heart and Circulatory Physiology, 302(6), pp.H1250-H1260.

Fukamachi, S. et al., 2004. Conserved function of medaka pink-eyed dilution in melanin synthesis and its divergent transcriptional regulation in gonads among vertebrates. Genetics, 168(3), pp.1519-1527.

Grabher, C. \& Wittbrodt, J., 2007. Meganuclease and transposon mediated transgenesis in medaka. Genome biology, 8 Suppl 1(Suppl 1), p.S10.

Gianulis, E.C. \& Trudeau, M.C., 2011. Rescue of Aberrant Gating by a Genetically Encoded PAS (Per-Arnt-Sim) Domain in Several Long QT Syndrome Mutant Human Ether-á-gogo-related Gene Potassium Channels. Journal of Biological Chemistry, 286(25), pp.22160-22169.

Gierten, J. et al., 2020. Automated high-throughput heartbeat quantification in medaka and zebrafish embryos under physiological conditions. Scientific Reports, pp.1-12.

Gillum, R.F., Makuc, D.M. \& Feldman, J.J., 1991. Pulse rate, coronary heart disease, and death: The NHANES I Epidemiologic Follow-up Study. American Heart Journal, 121(1), pp.172-177.

Gut, P. et al., 2017. Little Fish, Big Data: Zebrafish as a Model for Cardiovascular and Metabolic Disease. Physiological Reviews, 97(3), pp.889-938.

Hammouda, O.T. et al., 2019. Swift Large-scale Examination of Directed Genome Editing S. C. F. Neuhauss, ed. PLoS ONE, 14(3), pp.e0213317-11. 
Han, P. et al., 2014. Hydrogen peroxide primes heart regeneration with a derepression mechanism. Nature Publishing Group, pp.1-17.

Heyde, von der, B. et al., 2020. Translating GWAS-identified loci for cardiac rhythm and rate using an in vivo image- and CRISPR/Cas9-based approach. Scientific Reports, pp.1-18.

Hiura, Y. et al., 2010. A Genome-Wide Association Study of Hypertension-Related Phenotypes in a Japanese Population. Circulation Journal, 74(11), pp.2353-2359.

Hoed, den, M. et al., 2013. Identification of heart rate-associated loci and their effects on cardiac conduction and rhythm disorders. Nature Genetics, pp.1-14.

Holm, H. et al., 2011. A rare variant in MYH6 is associated with high risk of sick sinus syndrome. Nature Genetics, 43(4), pp.316-320.

$\mathrm{Hu}, \mathrm{Z}$. et al., 2013. A genome-wide association study identifies two risk loci for congenital heart malformations in Han Chinese populations. Nature Genetics, pp.1-5.

Huttner, I.G. et al., 2013. A transgenic zebrafish model of a human cardiac sodium channel mutation exhibits bradycardia, conduction-system abnormalities and early death. Journal of Molecular and Cellular Cardiology, 61(C), pp.123-132.

Ikram, M.A. et al., 2009. Genomewide Association Studies of Stroke. New England Journal of Medicine, 360(17), pp.1718-1728.

Itoh-Satoh, M. et al., 2002. Titin Mutations as the Molecular Basis for Dilated Cardiomyopathy. Biochemical and Biophysical Research Communications, 291(2), pp.385-393.

Iwamatsu, T., 2004. Stages of normal development in the medaka Oryzias latipes. Mechanisms of Development, 121(7-8), pp.605-618.

Kathiresan, S. \& Srivastava, D., 2012. Genetics of Human Cardiovascular Disease. Cell, 148(6), pp.1242-1257.

Koster, R. et al., 1997. Medaka spalt acts as a target gene of hedgehog signaling. Development, 124(16), p.3147.

Lessman, C.A., 2011. The developing zebrafish (Danio rerio): A vertebrate model for highthroughput screening of chemical libraries R. S. Tuan, ed. Birth Defects Research Part C: Embryo Today: Reviews, 93(3), pp.268-280.

Li, J. et al., 2017. Essential role of Cdc42 in cardiomyocyte proliferation and cell-cell adhesion during heart development. Developmental Biology, 421(2), pp.271-283.

Lischik, C.Q., Adelmann, L. \& Wittbrodt, J., 2019. Enhanced in vivo-imaging in medaka by optimized anaesthesia, fluorescent protein selection and removal of pigmentation $\mathrm{C}$. Winkler, ed. PLoS ONE, 14(3), pp.e0212956-19. 
Marroni, F. et al., 2009. A Genome-Wide Association Scan of RR and QT Interval Duration in 3 European Genetically Isolated Populations. Circulation: Cardiovascular Genetics, 2(4), pp.322-328.

Matsa, L.S. et al., 2014. Endothelin 1 gene as a modifier in dilated cardiomyopathy. Gene, 548(2), pp.256-262.

McGill, H.C., Jr, McMahan, C.A. \& Gidding, S.S., 2008. Preventing Heart Disease in the 21st Century. Circulation, 117(9), pp.1216-1227.

MD, P.M.B. et al., 2010. Heart rate as a risk factor in chronic heart failure (SHIFT): the association between heart rate and outcomes in a randomised placebo-controlled trial. The Lancet, 376(9744), pp.886-894.

Meyer, H.V. et al., 2020. Genetic and functional insights into the fractal structure of the heart. Nature, pp.1-25.

Nemtsas, P. et al., 2010. Adult zebrafish heart as a model for human heart? An electrophysiological study. Journal of Molecular and Cellular Cardiology, 48(1), pp.161-171.

Newton-Cheh, C. et al., 2009. Common variants at ten loci influence QT interval duration in the QTGEN Study. Nature Genetics, 41(4), pp.399-406.

Oxendine, S.L. et al., 2006. Adapting the medaka embryo assay to a high-throughput approach for developmental toxicity testing. NeuroToxicology, 27(5), pp.840-845.

Paré, G. et al., 2013. Genetic Determinants of Dabigatran Plasma Levels and Their Relation to Bleeding. Circulation, 127(13), pp.1404-1412.

Park, C.Y. et al., 2010. skNAC, a Smyd1-interacting transcription factor, is involved in cardiac development and skeletal muscle growth and regeneration. Proc Natl Acad Sci USA, 107(48), p.20750.

Pfeufer, A. et al., 2009. Common variants at ten loci modulate the QT interval duration in the QTSCD Study. Nature Genetics, 41(4), pp.407-414.

Qian, L. et al., 2011. Tinman/Nkx2-5 acts via miR-1 and upstream of Cdc42 to regulate heart function across species. The Journal of Cell Biology, 193(7), pp.1181-1196.

R Core Team (2019). R: A language and environment for statistical computing. R Foundation for Statistical Computing, Vienna, Austria. URL https://www.R-project.org/

Rathjens, F.S. et al., 2020. Preclinical evidence for the therapeutic value of TBX5 normalization in arrhythmia control. Cardiovascular Research.

Rembold, M. et al., 2006. Transgenesis in fish: efficient selection of transgenic fish by coinjection with a fluorescent reporter construct. Nature Protocols, 1(3), pp.1133-1139. 27 
Ritchie, M.D. et al., 2013. Genome- and Phenome-Wide Analyses of Cardiac Conduction Identifies Markers of Arrhythmia Risk. Circulation, 127(13), pp.1377-1385.

RStudio Team (2018). RStudio: Integrated Development for R. RStudio, Inc., Boston, MA URL http://www.rstudio.com/

Shankaran, S.S. et al., 2018. CRISPR/Cas9-Directed Gene Editing for the Generation of Lossof-Function Mutants in High-Throughput Zebrafish F 0Screens. Current Protocols in Molecular Biology, 119(1), pp.4257-22.

Smith, J.G. et al., 2009. Genome-wide association study of electrocardiographic conduction measures in an isolated founder population: Kosrae. HRTHM, 6(5), pp.634-641.

Sotoodehnia, N. et al., 2010. Common variants in 22 loci are associated with QRS duration and cardiac ventricular conduction. Nature Genetics, pp.1-11.

Stemmer, M. et al., 2015. CCTop: An Intuitive, Flexible and Reliable CRISPR/Cas9 Target Prediction Tool S. Maas, ed. PLoS ONE, 10(4), pp.e0124633-11.

Targoff, K.L. et al., 2013. Nkx genes are essential for maintenance of ventricular identity. Development, 140(20), pp.4203-4213.

Teboul, L., 2017. Phenotyping first-generation genome editing mutants: a new standard? Mammalian Genome, 28(7), pp.377-382.

Thanassoulis, G. et al., 2013. Genetic Associations with Valvular Calcification and Aortic Stenosis. New England Journal of Medicine, 368(6), pp.503-512.

Thumberger, T. et al., 2021. hei-tag: a highly efficient tag to boost targeted genome editing. bioRxiv, p.2021.05.27.445956.

Vasan, R.S. et al., 2009. Genetic Variants Associated With Cardiac Structure and Function: A Meta-analysis and Replication of Genome-wide Association Data. JAMA, 302(2), pp.168-178.

Villard, E. et al., 2011. A genome-wide association study identifies two loci associated with heart failure due to dilated cardiomyopathy. European Heart Journal, 32(9), pp.10651076.

Wickham, H., 2016. ggplot2: Elegant Graphics for Data Analysis, Springer-Verlag New York. Available at: https://ggplot2.tidyverse.org.

Wittbrodt, J., Shima, A. \& Schartl, M., 2002. MEDAKA - A MODEL ORGANISM FROM THE FAR EAST. Nature Reviews Genetics, 3(1), pp.53-64.

Wooten, E.C. et al., 2010. Application of Gene Network Analysis Techniques Identifies AXIN1/PDIA2 and Endoglin Haplotypes Associated with Bicuspid Aortic Valve A. E. Toland, ed. PLoS ONE, 5(1), pp.e8830-10. 28 
612 Wu, R.S. et al., 2018. A Rapid Method for Directed Gene Knockout for Screening in G0

613 Zebrafish. Developmental Cell, 46(1), pp.112-125.e4.

614 Yamaguchi, T. et al., 2018. The CCR4-NOT deadenylase complex controls Atg7-dependent cell death and heart function. Sci. Signal., 11(516), p.eaan3638.

616 Yates, A.D. et al., 2020. Ensembl 2020. Nucleic Acids Research, 48(D1), pp.D682-D688.

617 Yonekura, M. et al., 2018. Medaka as a model for ECG analysis and the effect of verapamil.

618 Journal of Pharmacological Science, 137(1), pp.55-60.

619 Zakariyah, A.F. et al., 2017. Congenital heart defect causing mutation in Nkx2.5 displays in vivo functional deficit. Journal of Molecular and Cellular Cardiology, 105(C), pp.89-98.

Zaklyazminskaya, E. \& Dzemeshkevich, S., 2016. The role of mutations in the SCN5A gene in cardiomyopathies. BBA - Molecular Cell Research, 1863(Part B), pp.1799-1805.

623

624 
bioRxiv preprint doi: https://doi.org/10.1101/2021.02.03.429563; this version posted June 21, 2021. The copyright holder for this preprint (which was not certified by peer review) is the author/funder, who has granted bioRxiv a license to display the preprint in perpetuity. It is made available under aCC-BY-NC-ND 4.0 International license.
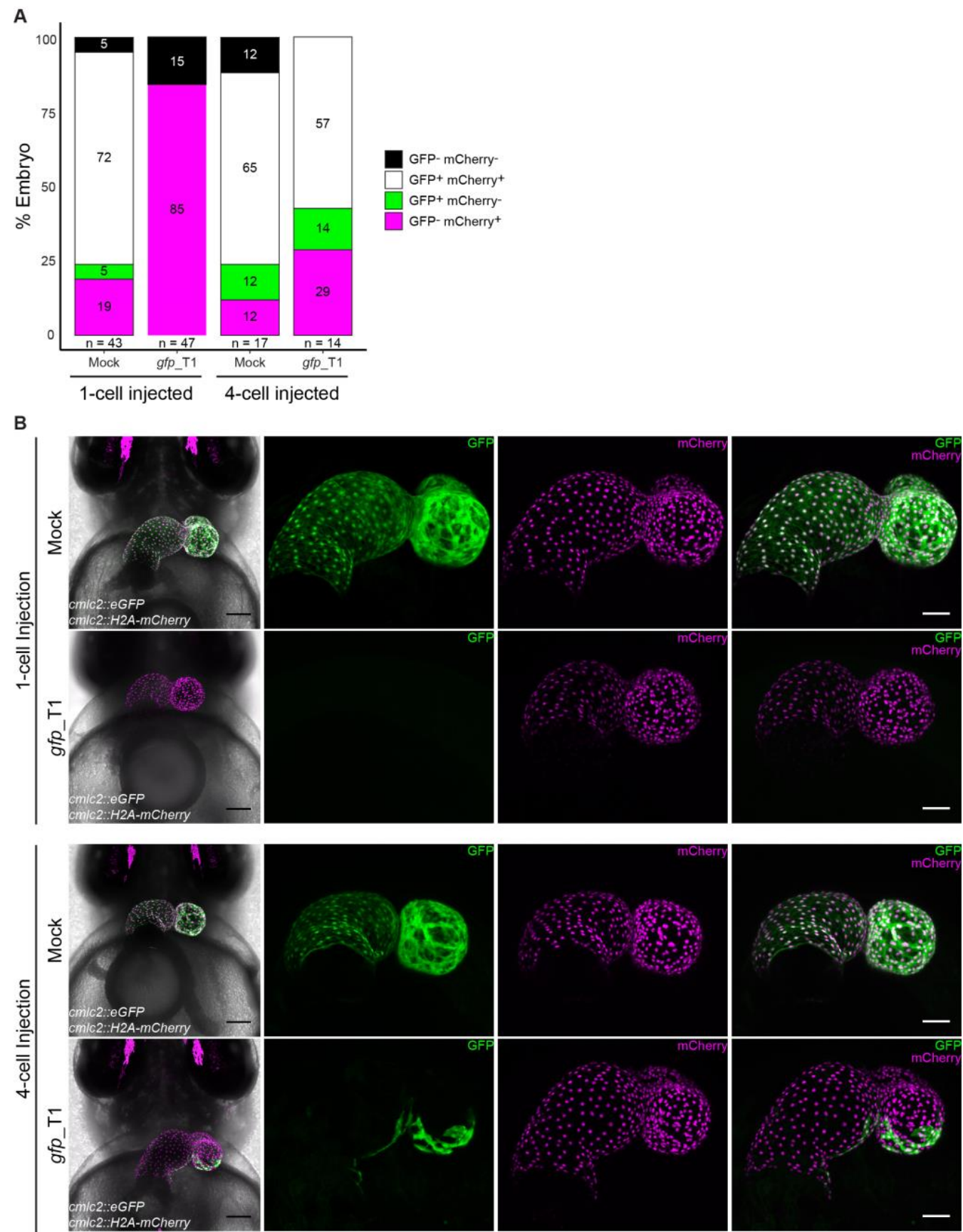

Figure S1. Highly efficient CRISPR/Cas9-mediated editing in injected (F0) generation

A Distribution of reporter expression in mock injected and $g f p \_T 1$ crispants of the $c m l c 2:: e G F P$ cmlc2::H2A-mCherry reporter line (4 dpf). Embryos were injected either at the 1-cell or 4-cell stage. Note: complete lack of GFP-expressing embryos when injected at the 1-cell stage. Biological replicates for each group is denoted (n). B Confocal images of GFP expression in mock-injected and $g f p$ crispant embryo hearts of the $c m l c 2:: e G F P$ cmlc $2:: H 2 A-m$ Cherry reporter line (7 dpf). Embryos were injected either at the 1-cell or 4-cell stage. Note: complete loss of GFP expression when injected at the 1-cell stage $(n=8 / 8)$, while mosaic expression when injected at 4-cell stage $(n=4 / 4)$. Scale bars: $100 \mu \mathrm{m}$ (First panel on left) and $50 \mu \mathrm{m}$ (blow-up images). 
bioRxiv preprint doi: https://doi.org/10.1101/2021.02.03.429563; this version posted June 21, 2021. The copyright holder for this preprint (which was not certified by peer review) is the author/funder, who has granted bioRxiv a license to display the preprint in perpetuity. It is made available under aCC-BY-NC-ND 4.0 International license.
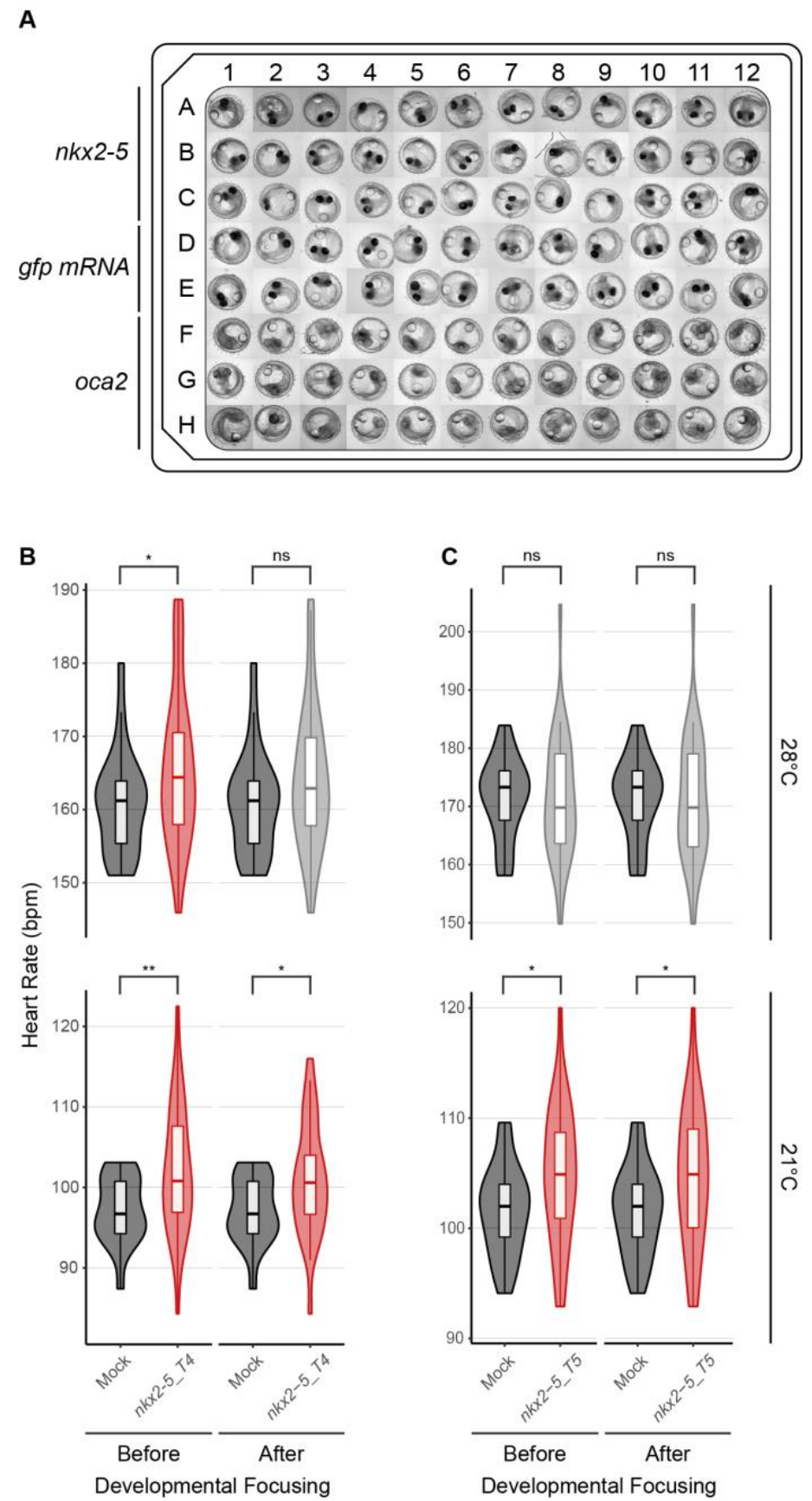

Figure S2. Consistent heart rate phenotype observed in medaka $n k x 2-5$ crispants

A Overview of 96-well plate with embryos (4 dpf) injected with sgRNA against $n k x 2-5$ or oca2, as well as embryos mock injected with GFP $m R N A$ (Fig 1D). Note the loss of eye pigmentation in oca2 crispant embryos. B-C Heart rate measurements of GFP-injected (Mock; dark grey) and $n k x 2-5$ crispant embryos (4 dpf) (B second replicate of $n x k 2-5 \_T 4$; $\mathbf{C}$ different sgRNA $n k x 2-5 \_T 5$ targeting same region of interest) at 21 and $28^{\circ} \mathrm{C}$, before and after exclusion of severely affected embryos (< stage 28; developmental focusing). Significant differences are shown in red and were determined by two-tailed Student's t-test; ${ }^{*} p<0.05,{ }^{* *} p<0.01$, ns (not significant; light grey). For biological replicates see Source Data Fig S2. 


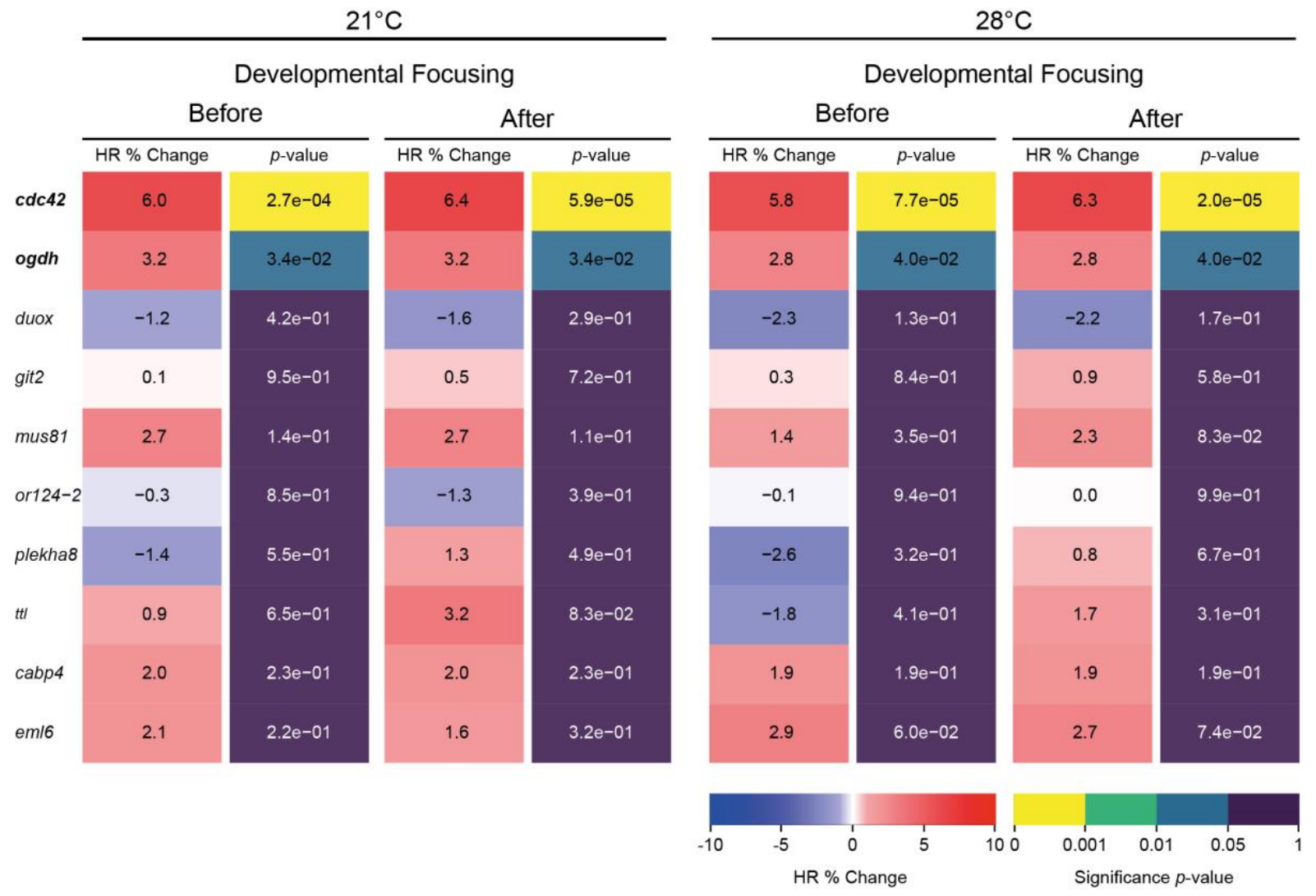

Figure S3. Developmental focusing does not alter analysis outcome of random gene selection

Heatmap quantitative representation of the comparative heart rate analysis between each crispant embryo group and its corresponding control sibling group before and after developmental focusing; for each measured temperature, the percent change in mean heart rate (HR \% Change) between crispants and their corresponding control sibling, flanked by the statistical significance ( $p$-value) of the observed change calculated by two-tailed Student's t-test on the full distribution. Genes showing significant heart rate phenotypes are indicated in bold. For biological replicates see Source Data Fig S3. 

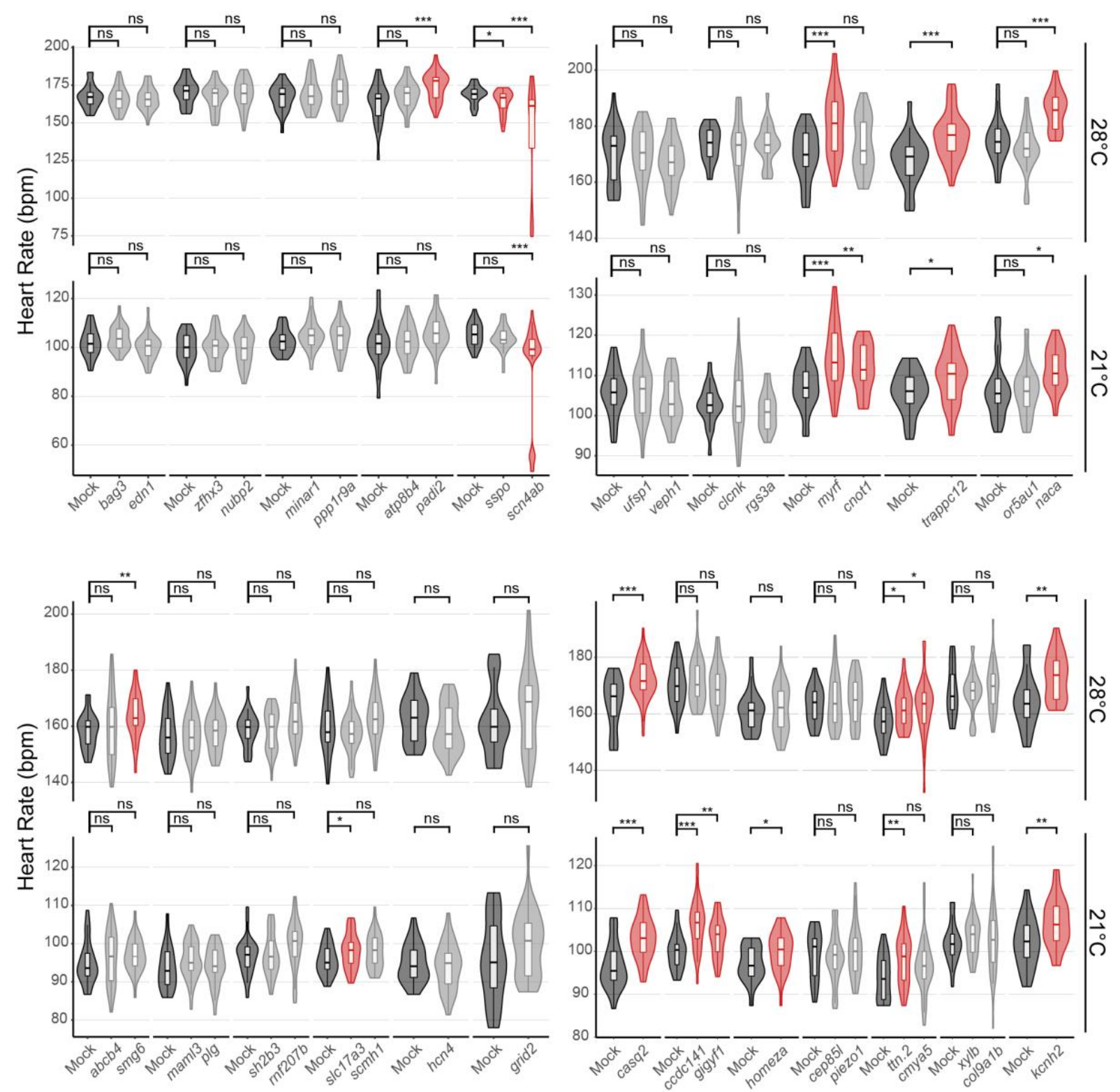

Figure S4. Comparative analysis of mean heart rates in targeted hGWAS gene selection

Heart rate measurements (beats per minute, bpm) of GFP-injected (Mock; dark grey) and corresponding sibling crispant embryos (4 dpf) at 21 and $28^{\circ} \mathrm{C}$ after developmental focusing (also see heatmap representation of the data in Fig 3A). Different experimental plates are represented by breaks on the $\mathrm{x}$ axis. Significant differences in mean heart rates were determined between each crispant embryo group and its corresponding sibling control group by two-tailed Student's t-test; $* p<0.05, * * p<0.01, * * * p$ $<0.001$, ns (not significant). Red groups correspond to crispants showing significant heart rate phenotypes, and light grey groups correspond to crispants showing no significant heart rate phenotype. For biological replicates see Source Data Fig S4. 
bioRxiv preprint doi: https://doi.org/10.1101/2021.02.03.429563; this version posted June 21,2021 . The copyright holder for this preprint

(which was not certified by peer review) is the author/funder, who has granted bioRxiv a license to display the preprint in perpetuity. It is made available under aCC-BY-NC-ND 4.0 International license.

$21^{\circ} \mathrm{C}$

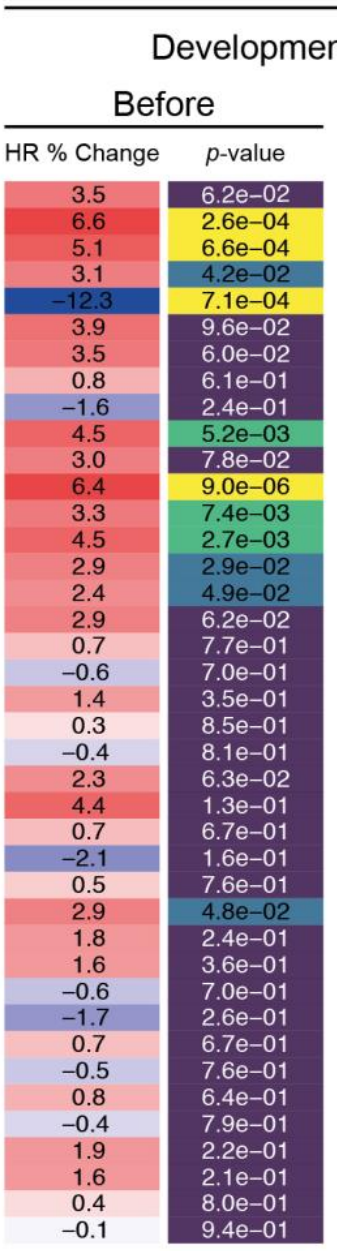

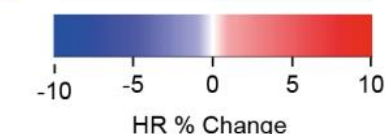

After
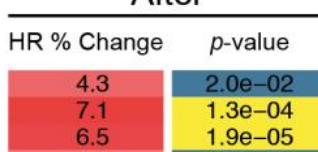

\begin{tabular}{c|c|c}
-12.3 & $7.1 \mathrm{e}-04$ \\
3.9 & $9.6 \mathrm{e}-02$ \\
4.5 & $6.6 e-03$
\end{tabular}

\begin{tabular}{l|l|}
4.5 & $6.6 \mathrm{e}-03$ \\
2.1 & $1.6 \mathrm{e}-01$ \\
-1.6 & $2.4 \mathrm{e}-01$
\end{tabular}

2.1
-1.6

-1.6
4.5

$3.0 \quad 5.2 \mathrm{e}-03$

\begin{tabular}{l|l}
6.4 & $7.8 \mathrm{e}-02$ \\
\hline 3.3 & $9.0 \mathrm{e}-06$
\end{tabular}

$\begin{array}{ll}3.3 & 7.4 \mathrm{e}-03 \\ 4.4 & 9.8 \mathrm{e}-03\end{array}$

2.9
2.4

$3.0 \quad 6.2 \mathrm{e}-02$

$0.4 \quad 8.5 \mathrm{e}-01$

$-0.9 \quad 5.6 \mathrm{e}-01$

$\begin{array}{ll}1.4 & 3.5 e-01 \\ 0.3 & 8.5 e-01\end{array}$

$-0.4 \quad 8.1 \mathrm{e}-01$

$2.3 \quad 6.3 e-02$

4.4
0.7

$-2.1 \quad 1.6 e-01$

$0.5 \quad 7.6 \mathrm{e}-01$

$1.6 \quad 3.6 \mathrm{e}-01$

$-0.6 \quad 7.0 \mathrm{e}-01$

\begin{tabular}{r|r}
-1.7 & $2.6 e-01$ \\
0.7 & $6.3 e-01$
\end{tabular}

$-0.5 \quad 7.6 \mathrm{e}-01$

$0.7 \quad 6.7 \mathrm{e}-01$

$-1.3 \quad 3.7 e-01$

1.6 2.1e-0

\begin{tabular}{r|r}
0.4 & $8.0 \mathrm{e}-01$ \\
-0.1 & $9.4 \mathrm{e}-01$
\end{tabular}

HR \% Change

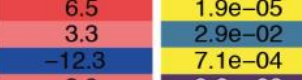

\begin{tabular}{l|l}
4.4 & $9.8 \mathrm{e}-03$ \\
2.9 & $2.9 \mathrm{e}-02$
\end{tabular}

\begin{tabular}{l|l}
2.4 & $1.1 \mathrm{e}-01$ \\
1.8 & $2.4 \mathrm{e}-01$
\end{tabular}

$2.1 \quad 1.7 e-01$ $28^{\circ} \mathrm{C}$

Developmental Focusing

\begin{tabular}{|c|c|c|c|}
\hline \multicolumn{2}{|c|}{ Before } & \multicolumn{2}{|c|}{ After } \\
\hline HR \% Change & $p$-value & HR \% Change & $p$-value \\
\hline 4.5 & $3.4 \mathrm{e}-03$ & 6.2 & $1.0 e-05$ \\
\hline 6.5 & $1.8 \mathrm{e}-04$ & 6.8 & $1.3 e-04$ \\
\hline 3.6 & $1.7 e-02$ & 5.1 & $4.6 e-04$ \\
\hline 5.0 & $7.1 \mathrm{e}-04$ & 5.2 & $4.7 e-04$ \\
\hline-15.2 & $5.7 e-04$ & -15.2 & $5.7 e-04$ \\
\hline 7.1 & $8.3 e-04$ & 7.1 & $8.3 e-04$ \\
\hline 5.2 & $1.5 e-03$ & 5.2 & $1.5 \mathrm{e}-03$ \\
\hline 2.1 & $1.2 \mathrm{e}-01$ & 3.4 & $9.7 e-03$ \\
\hline-2.9 & $1.3 e-02$ & -2.9 & $1.3 e-02$ \\
\hline 2.7 & $2.5 e-02$ & 2.7 & $2.5 e-02$ \\
\hline 3.1 & $3.0 \mathrm{e}-02$ & 3.1 & $3.0 \mathrm{e}-02$ \\
\hline 0.8 & $5.2 e-01$ & 0.8 & $5.2 \mathrm{e}-01$ \\
\hline-0.5 & $6.9 \mathrm{e}-01$ & -0.5 & $6.9 e-01$ \\
\hline 2.7 & $6.2 \mathrm{e}-02$ & 2.2 & $2.1 \mathrm{e}-01$ \\
\hline 0.5 & $7.2 \mathrm{e}-01$ & 0.5 & $7.2 \mathrm{e}-01$ \\
\hline-1.4 & $3.4 e-01$ & -1.4 & $3.4 \mathrm{e}-01$ \\
\hline 2.2 & $6.7 e-02$ & 2.3 & $6.4 \mathrm{e}-02$ \\
\hline 3.3 & $9.8 \mathrm{e}-02$ & 3.6 & $8.1 \mathrm{e}-02$ \\
\hline-2.2 & $1.1 \mathrm{e}-01$ & -2.3 & $1.0 \mathrm{e}-01$ \\
\hline 2.5 & $1.4 \mathrm{e}-01$ & 2.5 & $1.4 \mathrm{e}-01$ \\
\hline-1.9 & $1.7 e-01$ & -1.9 & $1.7 e-01$ \\
\hline-1.7 & $1.9 e-01$ & -1.7 & $1.9 e-01$ \\
\hline 1.9 & $2.4 \mathrm{e}-01$ & 1.9 & $2.4 \mathrm{e}-01$ \\
\hline 2.6 & $2.7 e-01$ & 2.6 & $2.7 e-01$ \\
\hline 1.2 & $3.1 \mathrm{e}-01$ & 1.2 & $3.1 \mathrm{e}-01$ \\
\hline-1.5 & $3.2 e-01$ & -1.5 & $3.2 \mathrm{e}-01$ \\
\hline-1.2 & $3.8 \mathrm{e}-01$ & -1.2 & $3.8 e-01$ \\
\hline 2.1 & $2.0 e-01$ & 1.2 & $4.4 e-01$ \\
\hline-0.9 & $4.9 \mathrm{e}-01$ & -0.9 & $4.9 e-01$ \\
\hline 1.1 & $4.9 e-01$ & 1.1 & $4.9 e-01$ \\
\hline 0.8 & $5.2 e-01$ & 0.8 & $5.2 \mathrm{e}-01$ \\
\hline-0.7 & $5.6 e-01$ & -0.7 & $5.6 e-01$ \\
\hline 0.9 & $5.2 e-01$ & 0.8 & $5.7 e-01$ \\
\hline-0.8 & $5.9 e-01$ & -0.8 & $5.9 e-01$ \\
\hline 0.8 & $5.8 e-01$ & 0.7 & $6.0 \mathrm{e}-01$ \\
\hline 0.4 & $7.3 e-01$ & -0.2 & $9.0 e-01$ \\
\hline-0.4 & $7.9 e-01$ & -0.1 & $9.2 \mathrm{e}-01$ \\
\hline-0.1 & $9.3 e-01$ & -0.1 & $9.3 e-01$ \\
\hline-0.1 & $9.4 \mathrm{e}-01$ & -0.1 & $9.4 \mathrm{e}-01$ \\
\hline-0.1 & $9.6 e-01$ & -0.1 & $9.6 \mathrm{e}-01$ \\
\hline
\end{tabular}

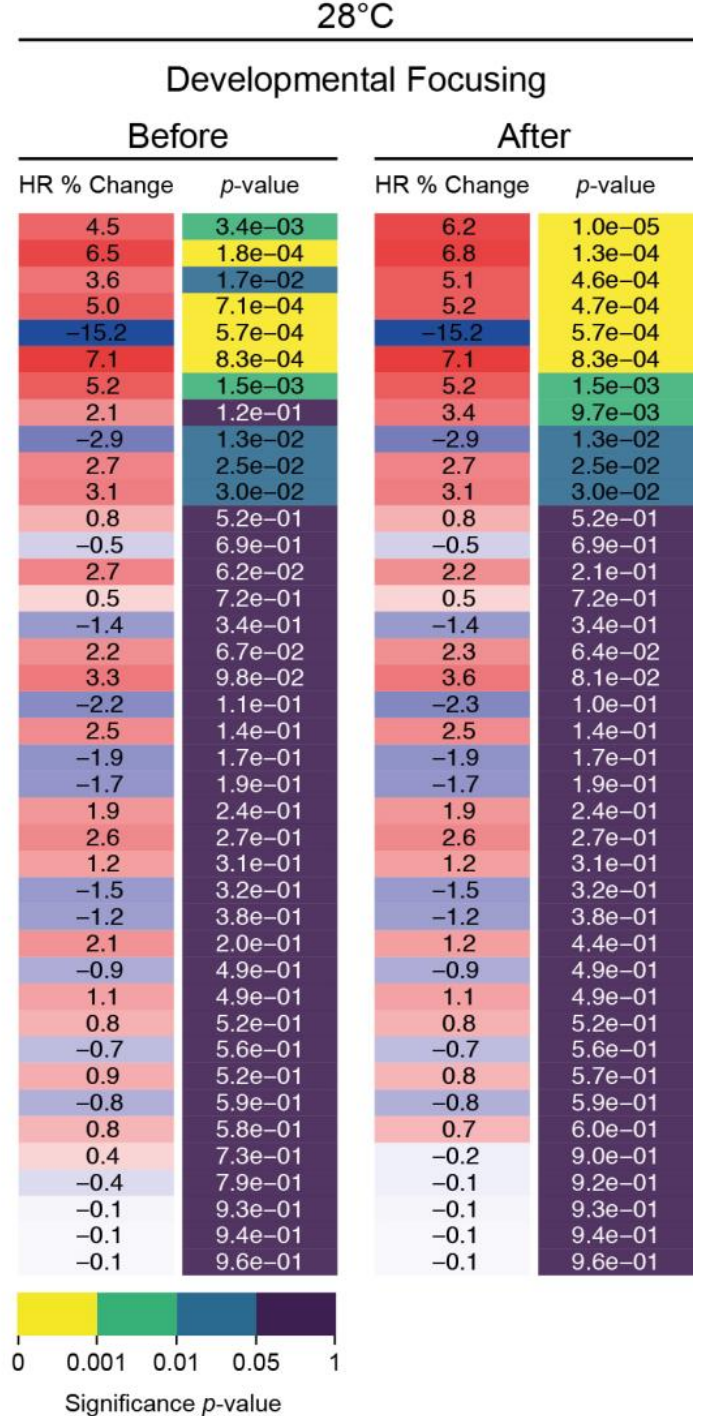

Fig S5. Developmental focusing does not alter analysis outcome of targeted hGWAS genes

Heatmap quantitative representation of the comparative heart rate analysis between each crispant embryo group and its corresponding control sibling group before and after developmental focusing; for each measured temperature, the percent change in mean heart rate (HR \% Change) between crispants and their corresponding control siblings, flanked by the statistical significance ( $p$-value) of the observed change, calculated by two-tailed Student's t-test on the full distribution. Genes showing significantly different heart rate phenotypes are indicated in bold. For biological replicates see Source Data Fig S4. 
630 Movie S1. Moderate AV-block arrhythmia observed in medaka Fo scn4ab crispants and 631 homozygous F2 mutants

632 Side by side comparison of rhythmic heartbeat of GFP-injected (Mock; left) and arrhythmic scn $4 a b$ 633 crispants (F0; middle) as well as homozygous mutants (F2; right) displaying 2:1 AV-block phenotype. 634 Videos of medaka embryos $(5 \mathrm{dpf})$ were acquired using a stereomicroscope under bright field 635 illumination.

Movie S2. Severe AV-block arrhythmia observed in medaka scn4ab crispants

Side by side comparison of rhythmic heartbeat of GFP-injected (Mock; left) and arrhythmic scn4ab crispants displaying severe AV-block phenotype (right). Videos of medaka embryos (9 dpf) were acquired using a stereomicroscope under bright field illumination.

641

Table S1. List of sgRNAs used

\begin{tabular}{|c|c|}
\hline sgRNA & Target sequence [PAM] \\
\hline abcb4_T7 & ATGTCCCTGAGTGTGAAGAG[TGG] \\
\hline atp8b4_T2 & TAAGTTCTACGATAACACCC[TGG] \\
\hline bag3_T1 & GAGCAGGGCGCCGTTCACCC[GGG] \\
\hline cabp4_T9 & GAATTTGACTACGATGCAGA[TGG] \\
\hline casq2_T1 & TGTTGTCTGGGATGGGTTCG[TGG] \\
\hline ccdc141_T1 & CCAGGAACCTGAAGGTTGTC[AGG] \\
\hline$c d c 42 \_T 2$ & AGAGCGGAGAGAAACTGGCT[CGG] \\
\hline cep85l_T5 & AACCCAGGACTTCTCAGATA[GGG] \\
\hline clcnk_T10 & TTCTCCACTGGAGTAGTTTT[TGG] \\
\hline cmya5_T1 & GTAGAACAGGTAATTCTCGT[TGG] \\
\hline cnot1_T5 & GCCAAGTGTTGACAATACTG[AGG] \\
\hline col9alb_T2 & GACGGACGCGTAGGCATTCC[AGG] \\
\hline duox_T3 & CATTCGGCACGCTTTCTCTA[AGG] \\
\hline edn1_T4 & GCCGACGGAGTCTGCGCGGA[GGG] \\
\hline eml6_T2 & CTGCGCTGTTCGCACGCTAA[AGG] \\
\hline$g f p \_T l$ & AGCACTGCACGCCGTAGGTC[AGG] \\
\hline gigyf1_T3 & GAATGAACCGGCATGAACGC[CGG] \\
\hline git2_T3 & TAAACGCCTTCGAAACACGG[AGG] \\
\hline grid2_T8 & AAAGGCTACGGCTAAGGGTC[TGG] \\
\hline hcn4_T5 & AAACTCCCTTCGAACTTGTG[AGG] \\
\hline homeza_T1 & GCTACCAGCAGGTGCGAGAT[TGG] \\
\hline kcnh2_T1 & CATCACTGCTGGGAGAACCG[GGG] \\
\hline maml3_T19 & TCATGTAAGGTGTCATCATA[GGG] \\
\hline
\end{tabular}




\begin{tabular}{|c|c|}
\hline sgRN & arget sequence [PAM] \\
\hline & ТТССССТССССC \\
\hline & AGAGGATGGACGACCTCTG[TGG] \\
\hline & CTTATTGGAGTCCATATTG[TGG] \\
\hline naca_T1 & TGGTCTTAGGCAAGTAACG[GGG] \\
\hline$n k x 2-5 \_T 4$ & CCGCGGGTCCTCTTCTCCC[AGG] \\
\hline & CGGACAGACCCAAGCCCCGG[AGG \\
\hline & \\
\hline oca2_T3 & TGCAGGAATCATTCTGTGT[GGG] \\
\hline ogdh_T4 & AAAGCTGGACTTGGCCTCA[GGG] \\
\hline or124-2_T1 & GCCCACGTGGTCTCGTACGC[CGG] \\
\hline & GTCATGGACTCTTGCCTGTA[TGG] \\
\hline & \\
\hline piezol_T1 & TTGGGAGCCCGGATGTAGTT[AGG] \\
\hline plekha8_T9 & ICGGTGAAGCTTTCATAACA[CGG] \\
\hline plg_T1 & GGCAACGGGGCCAATTATCG[AGG] \\
\hline ppp1r9a_T7 & TGGTTCTTAGGAGATTCGGG[TGG] \\
\hline & GATCAAGTCACAGTCCAAGA[TGG] \\
\hline$r n f 207 b \_T 2$ & TAGACTTGTTTGTACTGATC[TGG] \\
\hline scmhl_T2 & TCTGACCTGCGCTGTACGAG[TGG] \\
\hline$s c n 4 a b \_T 2$ & TTAGGCTTAGCAATCCGCAA[CGG] \\
\hline sh2b3_T1 & AAGAAGTCCGTCGCTGCAAT[CGG] \\
\hline slc17a3_T5 & GTCTTGGCGCCGATTGTGAC[AGG] \\
\hline smg6_T2 & AAAGATAAAACC \\
\hline sspo_T4 & GTGCACCAAGTCATGTGGTT[GGG] \\
\hline trappc12_T2 & CAACACGCAGTGCCTCAAGC[TGG] \\
\hline$t t l \_T 1$ & GCTGGTAAATTACTACAGAG[GGG] \\
\hline$t t n .2 \_T 1$ & GAGCTAGCTGTCAAAGCCAT[GGG] \\
\hline ufsp1_T1 & GCTGGAGAGCATCGCAGTCC[AGG] \\
\hline veph1_T1 & GGCTCTGGTGGAGGTGTCCC[AGG] \\
\hline$x y l b \_T 1$ & TTTCCAGTGTCAGTACCTAC[AGG] \\
\hline zfhx3_T1 & AGCTGAGCGCACCCTGCCTG[AGG] \\
\hline
\end{tabular}

Hammouda et al., 2021 Validating understudied GWAS heart genes 
Table S2. List of primers used for genotyping by PCR

651

\begin{tabular}{|c|c|}
\hline Target Gene & Primers (5' - 3' direction) \\
\hline$a b c b 4 L f$ & CCTCTGCAGAAGCTGGATGG \\
\hline$a b c b 4 \mathrm{Lr}$ & TTTGCGATGGCGTGTGAGCG \\
\hline atp8b4 Lf & ACCTACAGTCAAAGGGAAATGAGC \\
\hline atp8b4 Lr & ACGCTGCCTCGTCTGGTGAC \\
\hline bag3 Lf & CTTCGCTTAGGAGCCAGTCC \\
\hline bag3 $\mathrm{Lr}$ & CAGTATGGTCTGAACCCGCC \\
\hline cabp4 Lf & GTGCCATCTTGCCATGCTGC \\
\hline cabp4 Lr & ATCCTGCTGCTCCTGGTCCC \\
\hline casq2 Lf & CGAACAGGTCAAACCGTGTG \\
\hline casq2 Lr & GGAACTTGCACAAACGGACC \\
\hline$c c d c 141 \mathrm{Lf}$ & GGCTACGGATGAAGGAGCTC \\
\hline$c c d c 141 \mathrm{Lr}$ & TCCTGGCTAAGTGAAGCAGC \\
\hline$c d c 42 L f$ & TCCAGAGCTGCGAGGATGGC \\
\hline$c d c 42 \mathrm{Lr}$ & CCAGCCGCTCTGTTCTGGGC \\
\hline cep85l Lf & AGGAGTGAGCTGAGGATGGG \\
\hline cep85l Lr & GCATCCTCACTCTAGCCGGG \\
\hline clcnk $L f$ & GATTCATGCTGTGTTCCCGC \\
\hline clcnk Lr & CCACTGAGTTGGTCGCTTTG \\
\hline cmya5 Lf & TTTGTGGGAGCTCGTCTGGG \\
\hline cmya5 Lr & GCCACGCTGAGATGGAGCCC \\
\hline cnotl Lf & CCGGTTGTGGCGCCTTGAGG \\
\hline cnotl Lr & GCTTTGGGTCTGGCACTGCG \\
\hline col9alb Lf & AGGGACTCACTTACCGGAAGCCC \\
\hline col9alb Lr & CTGGTCCTCAGGGAGTGGCAGG \\
\hline duox $L f$ & GACGCTGTCAGCTCGCACTG \\
\hline duox $L r$ & GGTCCACTCATCGCTCCCTC \\
\hline$e d n 1 L f$ & TCTCCGTGCTGTCAGTGTTC \\
\hline$e d n 1 L r$ & ATGCTGGTTGCCATGGAGTC \\
\hline eml6 Lf & ACGCCACGGACAGCATGGTC \\
\hline eml6 Lr & TCACTGACAGCTTTGAAGGTTGACG \\
\hline$g f p L f$ & GTGAGCAAGGGCGAGGAGCT \\
\hline$g f p L r$ & CTTGTACAGCTCGTCCATGC \\
\hline gigyfl Lf & ACAGGAGATAGCAGCAGCAG \\
\hline
\end{tabular}




\begin{tabular}{|c|c|}
\hline Target Gene & Primers (5' - 3' direction) \\
\hline gigyfl Lr & CGTCCCTGATTGTAGATTTGCG \\
\hline git2 Lf & TTGCTGTCGCCGCTGCTCTG \\
\hline git $2 \mathrm{Lr}$ & GGGCTCATGGGACCTTATGTTGG \\
\hline grid2 Lf & ACCTACAGACCTGTAACTCCTTCGC \\
\hline grid2 $\mathrm{Lr}$ & ACCTGGAGCACCTACACCACAGG \\
\hline hcn4 Lf & GACCCGTCAAGTGCCACAGG \\
\hline$h c n 4 \mathrm{Lr}$ & CACACCCTCCTTGTCCCTTG \\
\hline homeza Lf & GCCAAGGAAGACCAAAGAGC \\
\hline homeza Lr & TCACAAACCTCGCTCTAGGC \\
\hline$k c n h 2 L f$ & TCGCCCAGGCAAGTCCAACG \\
\hline kcnh2 Lr & ACCTCATCGCCACTTGAGTGTG \\
\hline maml3 Lf & CGGCAGCCATGTTGTCGTAC \\
\hline maml3 $\mathrm{Lr}$ & AAGCGGGTTATGTGTGCTGC \\
\hline minarl Lf & CGGTCAGCATGAACTAAGCTTCGG \\
\hline minarl Lr & TGGAGACGGACAGTGATTCCAGTGG \\
\hline mus81 Lf & GAAGACGACGAGACGGCCGG \\
\hline mus81 Lr & TGACCCGAACAGTACCTTTGTG \\
\hline$m y r f L f$ & GGTCCACCAACCGCCTGCC \\
\hline myrf $L r$ & AGCATCCTAGCATTGCAGCC \\
\hline naca $L f$ & TCGCTCTCCTCCTGTACTGTAGGG \\
\hline naca $L r$ & TCTGAGTCTACTGAAGCCAGCC \\
\hline$n k x 2-5$ lf & TAGCTTTGAGGCCTGCAGAC \\
\hline$n k x 2-5 l r$ & CGCATAGTTCGTGTTGCAGG \\
\hline nubp2 Lf & ACTGATGAGCCAGCCAACCC \\
\hline nubp2 Lr & TGGTCTGTGGCCACATGGTG \\
\hline oca2 Lf & GTTAAAACAGTTTCTTAAAAAGAACAGGA \\
\hline oca2 Lr & AGCAGAAGAAATGACTCAACATTTTG \\
\hline$o g d h L f$ & GAACACTCGTCTGGCCGGCC \\
\hline$o g d h L r$ & TACAGCAAGTCCCGCCCACG \\
\hline or124-2 Lf & TGAGGCTCAGACCCTCCTGG \\
\hline or124-2 Lr & ATGCGGTCCGGATGAGCTGG \\
\hline or5aul Lf & GCATTCAGCAGTTCTTCTGTCTTC \\
\hline or5aul Lr & GGCTTTGATAGATCTGTGTGGAAGC \\
\hline padi2 Lf & GCATGACACTACCTGAGAATGAAAC \\
\hline padi2 $\mathrm{Lr}$ & AGCCTCAGTCATCTCAGTAAAGC \\
\hline
\end{tabular}

38

Hammouda et al., 2021 Validating understudied GWAS heart genes 


\begin{tabular}{|c|c|}
\hline Target Gene & Primers (5' - 3' direction) \\
\hline piezol Lf & GCAGCAGCAGGAAGGTCAGG \\
\hline piezol $\mathrm{Lr}$ & CCGCTGCATTAGACCACTGC \\
\hline plekha8 Lf & CTGGCACCTTCCTGTCCAGC \\
\hline plekha8 Lr & ACGGAAGTCGCCCAAGTCCG \\
\hline plg Lf & CACAAACACAGCCGCACGCC \\
\hline plg $\operatorname{Lr}$ & GTGGGCGTGTCCGAGTCGAC \\
\hline ppplr9a Lf & CGAGGTCCAACCGAGGCAGC \\
\hline ppplr9a Lr & TCTCTGTCAGATCTGAGCCGGG \\
\hline rgs3a Lf & TGAGCTGTGTTCGCCCACTC \\
\hline $\operatorname{rgs} 3 a \mathrm{Lr}$ & GGAGGGCTTCAACTGTGAGG \\
\hline$r n f 207 b L f$ & GCAGAAGCCTCCCATTGACG \\
\hline$r n f 207 b \mathrm{Lr}$ & CCTCTGGCTGACCGCTCCTG \\
\hline scmhl Lf & ACTCTGTTCGAGCGCCTCCC \\
\hline scmhl Lr & AGGCGCATCCTGCAGCTTCC \\
\hline $\operatorname{scn} 4 a b L f$ & TCACCACTTCTGCAGGATGG \\
\hline$s c n 4 a b \operatorname{Lr}$ & GAGGCGTAGTGATCTGACGG \\
\hline $\operatorname{sh} 2 b 3 L f$ & TTTCTGCCGGCATCTGCTCC \\
\hline $\operatorname{sh} 2 b 3 \mathrm{Lr}$ & CCTGCTGGTCGTTGTCCGTC \\
\hline slc17a3 Lf & CCGCTGTGCCCAGGAGCAAG \\
\hline slc17a3 Lr & AGGAAACGACCCGTGACCAC \\
\hline $\operatorname{smg6} L f$ & CCCAGCATCAGTGGAGGTGC \\
\hline smg6 Lr & CCCTGAGCCTCAGTCCCAGC \\
\hline sspo $L f$ & TGCAGTCGAGGGTCAGTGGTCG \\
\hline sspo Lr & CACAGGATCGCGGGCAAGAAGG \\
\hline trappc12 Lf & GTGCCTGCTGTACCTGGGCC \\
\hline trappc12 Lr & ACCACCACССТСТСТСССАGC \\
\hline ttl $L f$ & CCGGGCGGGACCAACAACTG \\
\hline$t t l \mathrm{Lr}$ & GGAGTCAGACAGCTCTGGGC \\
\hline $\operatorname{ttn} .2 L f$ & CAGAGCTGGACGGACATCGG \\
\hline $\operatorname{ttn} .2 \mathrm{Lr}$ & GGAGCGCTGACGTCTGCCTC \\
\hline ufspl Lf & TGGGAAAGGACTAGAGGAGGG \\
\hline$u f s p 1 L r$ & GACACTCGCTTCCAGGACAC \\
\hline vephl Lf & TCTGTCAGAGCGGATGCAAG \\
\hline veph1 $\mathrm{Lr}$ & AGTCTCTGATCGGGATGCAAC \\
\hline xylb Lf & CCTGCAGCAACATGGCAGTGTG \\
\hline
\end{tabular}

39

Hammouda et al., 2021 Validating understudied GWAS heart genes 
652

\begin{tabular}{|l|l|}
\hline Target Gene & Primers $(\mathbf{5}, \mathbf{- 3}$ ' direction) \\
\hline$x y l b L r$ & ACCATCACTGTGGTCAATGGCTGC \\
\hline$z f h x 3 L f$ & GGCTGGGTGGTGGGTGTCAG \\
\hline$z f h x 3 L r$ & TGTGCGGCTAGGTGGTGGAC \\
\hline
\end{tabular}

\section{Source Data Fig 1D. Biological replicates for Fig 1D}

Ns correspond to number of scored embryos per condition. before $\mathrm{DF}=$ before developmental focusing (i.e. all scored embryos). after DF $=$ after developmental focusing (i.e. only scored embryos looking older than stage 28)

\begin{tabular}{|l|c|c|c|c|c|c|}
\hline \multirow{2}{*}{ Gene_sgRNA } & \multicolumn{2}{|c|}{ Ns before DF } & \multicolumn{2}{c|}{ Ns after DF } & \multicolumn{2}{c|}{ Ns mock } \\
\cline { 2 - 6 } & 21C & 28C & 21C & 28C & 21C & 28C \\
\hline$n k x 2-5 \_T 4$ & 34 & 34 & 31 & 31 & \multirow{2}{*}{22} & \multirow{2}{*}{23} \\
\hline oca2_T1 & 35 & 35 & 35 & 35 & & \\
\hline
\end{tabular}

\section{Source Data Fig S2. Biological replicates for Fig S2 B-C}

Ns correspond to number of scored embryos per condition. before $\mathrm{DF}=$ before developmental focusing (i.e. all scored embryos). after DF $=$ after developmental focusing (i.e. only scored embryos looking older than stage 28)

\begin{tabular}{|l|l|l|l|l|l|l|}
\hline \multirow{2}{*}{ Gene_sgRNA } & \multicolumn{2}{|l|}{ Ns before DF } & \multicolumn{2}{l|}{ Ns after DF } & \multicolumn{2}{l|}{ Ns mock } \\
\cline { 2 - 7 } & 21C & 28C & 21C & 28C & 21C & 28C \\
\hline$n k x 2-5 \_T 4$ & 34 & 36 & 31 & 33 & 19 & 23 \\
\hline$n k x 2-5 \_T 5$ & 33 & 33 & 31 & 31 & 22 & 21 \\
\hline
\end{tabular}

\section{Source Data Fig S3. Biological replicates for Fig 2 and S3}

Ns correspond to number of scored embryos per condition. before DF = before developmental focusing (i.e. all scored embryos). after DF $=$ after developmental focusing (i.e. only scored embryos looking older than stage 28)

\begin{tabular}{|c|c|c|c|c|c|c|}
\hline \multirow[t]{2}{*}{ Gene } & \multicolumn{2}{|c|}{ Ns before DF } & \multicolumn{2}{|c|}{ Ns after DF } & \multicolumn{2}{|c|}{ Ns mock before -> after DF } \\
\hline & $21 \mathrm{C}$ & $28 \mathrm{C}$ & $21 \mathrm{C}$ & $28 \mathrm{C}$ & $21 \mathrm{C}$ & $28 \mathrm{C}$ \\
\hline$c d c 42$ & 32 & 32 & 29 & 30 & \multirow[t]{2}{*}{22} & \multirow[t]{2}{*}{20} \\
\hline ogdh & 31 & 33 & 31 & 33 & & \\
\hline duox & 32 & 33 & 27 & 28 & \multirow[t]{2}{*}{19} & \multirow[t]{2}{*}{21} \\
\hline git2 & 35 & 33 & 33 & 31 & & \\
\hline mus81 & 27 & 33 & 25 & 31 & \multirow[t]{2}{*}{$20->18$} & \multirow[t]{2}{*}{$21->20$} \\
\hline or124-2 & 34 & 34 & 30 & 30 & & \\
\hline plekha8 & 35 & 31 & 29 & 26 & \multirow[t]{2}{*}{21} & \multirow[t]{2}{*}{23} \\
\hline$t t l$ & 33 & 30 & 28 & 24 & & \\
\hline cabp4 & 34 & 34 & 34 & 34 & \multirow[t]{2}{*}{21} & \multirow[t]{2}{*}{21} \\
\hline eml6 & 36 & 34 & 34 & 32 & & \\
\hline
\end{tabular}

40 
Source Data Fig S4. Biological replicates for Fig 3A, S4 and S5

672

Ns correspond to number of scored embryos per condition. before $\mathrm{DF}=$ before developmental focusing

673 (i.e. all scored embryos). after DF $=$ after developmental focusing (i.e. only scored embryos looking 674 older than stage 28)

675

\begin{tabular}{|c|c|c|c|c|c|c|}
\hline \multirow[t]{2}{*}{ Gene } & \multicolumn{2}{|c|}{ Ns before DF } & \multicolumn{2}{|c|}{ Ns after DF } & \multicolumn{2}{|c|}{ Ns mock before -> after DF } \\
\hline & $21 \mathrm{C}$ & $28 \mathrm{C}$ & $21 \mathrm{C}$ & $28 \mathrm{C}$ & $21 \mathrm{C}$ & $28 \mathrm{C}$ \\
\hline bag3 & 32 & 33 & 32 & 33 & \multirow[t]{2}{*}{22} & \multirow[t]{2}{*}{24} \\
\hline$e d n 1$ & 35 & 34 & 35 & 34 & & \\
\hline$z f h x 3$ & 33 & 35 & 33 & 35 & \multirow[t]{2}{*}{23} & \multirow[t]{2}{*}{22} \\
\hline nubp2 & 36 & 34 & 36 & 34 & & \\
\hline minarl & 33 & 33 & 30 & 30 & \multirow[t]{2}{*}{24} & \multirow[t]{2}{*}{22} \\
\hline ppp1r9a & 32 & 34 & 32 & 34 & & \\
\hline$a t p 8 b 4$ & 35 & 34 & 32 & 31 & \multirow[t]{2}{*}{24} & \multirow[t]{2}{*}{23} \\
\hline padi2 & 31 & 36 & 31 & 36 & & \\
\hline sspo & 33 & 32 & 33 & 32 & \multirow[t]{2}{*}{22} & \multirow[t]{2}{*}{22} \\
\hline scn4ab & 35 & 32 & 35 & 32 & & \\
\hline ufspl & 33 & 32 & 33 & 32 & \multirow[t]{2}{*}{23} & \multirow[t]{2}{*}{24} \\
\hline veph1 & 34 & 35 & 34 & 35 & & \\
\hline clcnk & 34 & 31 & 34 & 31 & \multirow[t]{2}{*}{22} & \multirow[t]{2}{*}{23} \\
\hline rgs $3 a$ & 26 & 28 & 21 & 23 & & \\
\hline myrf & 34 & 34 & 33 & 33 & \multirow[t]{2}{*}{$24->23$} & \multirow[t]{2}{*}{$23->22$} \\
\hline cnotl & 36 & 35 & 22 & 22 & & \\
\hline trappc12 & 35 & 35 & 34 & 34 & 23 & 24 \\
\hline or5aul & 34 & 33 & 34 & 33 & \multirow[t]{2}{*}{22} & \multirow[t]{2}{*}{22} \\
\hline naca & 33 & 32 & 27 & 27 & & \\
\hline$a b c b 4$ & 36 & 35 & 36 & 35 & \multirow[t]{2}{*}{23} & \multirow[t]{2}{*}{22} \\
\hline smg6 & 36 & 35 & 30 & 29 & & \\
\hline maml3 & 35 & 35 & 34 & 34 & \multirow[t]{2}{*}{23} & \multirow[t]{2}{*}{24} \\
\hline plg & 35 & 36 & 33 & 34 & & \\
\hline $\operatorname{sh} 2 b 3$ & 35 & 36 & 35 & 36 & \multirow[t]{2}{*}{24} & \multirow[t]{2}{*}{24} \\
\hline$r n f 207 b$ & 35 & 36 & 33 & 34 & & \\
\hline slc17a3 & 36 & 35 & 36 & 35 & \multirow[t]{2}{*}{22} & 24 \\
\hline scmhl & 33 & 33 & 33 & 33 & & \\
\hline hcn4 & 35 & 36 & 33 & 34 & 23 & 24 \\
\hline grid2 & 30 & 34 & 30 & 34 & 23 & 20 \\
\hline casq2 & 59 & 56 & 53 & 50 & $25->24$ & $24->23$ \\
\hline ccdc141 & 36 & 34 & 36 & 34 & 22 & 23 \\
\hline gigyfl & 34 & 35 & 34 & 35 & & \\
\hline
\end{tabular}

41 
bioRxiv preprint doi: https://doi.org/10.1101/2021.02.03.429563; this version posted June 21,2021 . The copyright holder for this preprint (which was not certified by peer review) is the author/funder, who has granted bioRxiv a license to display the preprint in perpetuity. It is made available under aCC-BY-NC-ND 4.0 International license.

676

\begin{tabular}{|l|l|l|l|l|l|l|}
\hline \multirow{2}{*}{ Gene } & \multicolumn{2}{|l|}{ Ns before DF } & \multicolumn{2}{l|}{ Ns after DF } & \multicolumn{2}{l|}{ Ns mock before -> after DF } \\
\cline { 2 - 5 } & 21C & 28C & 21C & 28C & 21C & 28C \\
\hline homeza & 32 & 35 & 32 & 35 & 19 & 23 \\
\hline cep85l & 35 & 36 & 35 & 36 & 22 & 24 \\
\hline piezol & 35 & 33 & 35 & 33 & & \\
\hline ttn.2 & 34 & 35 & 34 & 35 & 23 & 24 \\
\hline cmya5 & 33 & 34 & 33 & 34 & & \\
\hline xylb & 35 & 33 & 35 & 33 & 23 & 22 \\
\hline col9a1b & 35 & 35 & 34 & 34 & & 21 \\
\hline kcnh2 & 34 & 36 & 34 & 36 & 21 -> 20 & 21 \\
\hline
\end{tabular}

